AEI-2011-024

MPP-2011-46

NSF-ITP-11-058

\title{
Explicit BCJ Numerators from Pure Spinors
}

\author{
Carlos R. Mafra ${ }^{a, b}$, Oliver Schlotterer ${ }^{b, c}$, and Stephan Stieberger ${ }^{b, c}$
}

a Max-Planck-Institut für Gravitationsphysik, Albert-Einstein-Institut, 14476 Potsdam, Germany

${ }^{b}$ Kavli Institute for Theoretical Physics, University of California, Santa Barbara, CA 93106, USA

${ }^{c}$ Max-Planck-Institut für Physik, Werner-Heisenberg-Institut, 80805 München, Germany

E-mails: crmafra@aei.mpg.de, olivers@mppmu.mpg.de, stephan.stieberger@mpp.mpg.de

\begin{abstract}
We derive local kinematic numerators for gauge theory tree amplitudes which manifestly satisfy Jacobi identities analogous to color factors. They naturally emerge from the low energy limit of superstring amplitudes computed with the pure spinor formalism. The manifestation of the color-kinematics duality is a consequence of the superstring computation involving no more than $(n-2)$ ! kinematic factors for the full color dressed $n$ point amplitude. The bosonic part of these results describe gluon scattering independent on the number of supersymmetries and captures any $\mathrm{N}^{k} \mathrm{MHV}$ helicity configuration after dimensional reduction to $D=4$ dimensions.
\end{abstract}




\section{Introduction}

Bern, Carrasco and Johansson (BCJ) have introduced a parametrization of gauge theory scattering amplitudes such that all the kinematic factors obey an equivalent of the Jacobi identity for color factors [1]. This duality between color- and kinematic degrees of freedom is an excellent example for hidden simplicity and non-obvious harmony in scattering amplitudes. It plays a crucial role for taming the non-planar sector of SYM and for understanding gravity as the double copy of gauge theories [2].

The BCJ organization scheme represents gauge theory amplitudes in terms of diagrams with cubic vertices only (in short: cubic diagrams). This amounts to writing the colordressed $n$ point amplitude as follows:

$$
\mathcal{A}_{n}=\sum_{i} \frac{c_{i} n_{i}}{\prod_{\alpha_{i}} s_{\alpha_{i}}}
$$

The $c_{i}$ denote color factors made of $n-2$ structure constants $f^{a b c}$ of the gauge group, and their dual numerators $n_{i}$ are constructed in this work. Each $\left(n_{i}, c_{i}\right)$ pair multiplies $n-3$ propagators $s_{\alpha_{i}}^{-1}$ of a cubic $n$ point tree diagram.

The contribution of four point vertices in SYM fields to (1.1) certainly contains less

propagators and must be absorbed into the $n_{i}$ by multiplying with some $\frac{s_{\alpha_{i}}}{s_{\alpha_{i}}}$ for compatibility with the pole structure. These contact terms introduce ambiguities in the parametrization above. At $n \geq 5$ points, a generic choice of assignment spoils the dual Jacobi identities for the $n_{i}$. Hence, contact terms have always been an obstacle in constructing color-dual BCJ numerators directly from the gauge theory. There exist Kawai-Lewellen-Tye (KLT) inspired expressions for $n_{i}$ in terms of color ordered gauge theory amplitudes [3,4] which do not exhibit manifest locality.

The approach used in this paper bypasses the contact term ambiguity because the new BRST cohomology organization of the string amplitude discussed in [5] naturally absorbs these contact terms. In $[6,7]$ the contact term ambiguity was shown to arise from the double pole in the OPE of two integrated vertices in the field-theory limit of the string amplitude. However, terms of this form are uniquely packaged inside BRST-covariant building blocks when the result of the tree-level string amplitude computed with the pure spinor formalism is recast into a form which manifests its BRST properties [5]. 
The $n_{i}$ constructed this way can therefore be recycled from planar $\mathcal{N}=4 \mathrm{SYM}$ to the non-planar sector and used for $\mathcal{N}=8$ supergravity by means of the double copy construction, cf. [8] and references therein. Ultimately, they are helpful for studying the ultraviolet properties of gravity theories at higher loops.

Due to the pure spinor methods, the BCJ numerators are written in terms of $\mathcal{N}=1$ SYM superfields in $D=10$ dimensions. It is straightforward to dimensionally reduce the superfield components to $D=4$, and the bosonic parts describe gluon scattering independently on the existence of supersymmetries. The computation does not single out any particular helicity configuration and treats all $\mathrm{N}^{k} \mathrm{MHV}$ amplitudes on the same footing.

\section{Basic facts of the Pure Spinor Formalism}

The explicit construction of BCJ numerators will be carried out in pure spinor superspace and we show the fine structure of the $(n-2)$ ! basic kinematics provided by the stringy computation of $n$-point amplitudes. For this purpose, this section briefly presents the tree-level framework of the pure spinor formalism $[9]^{1}$. In particular, we will make use of the pure spinor BRST cohomology building blocks which are more carefully explained in $[5]$.

The pure spinor formalism is a manifestly super-Poincare covariant approach to the superstring. Massless states of the open string sector are described by the ten-dimensional $\mathcal{N}=1$ super Yang-Mills superfields $\left[A_{\alpha}, A_{m}, W^{\alpha}, \mathcal{F}_{m n}\right]$ of [12] which encompass the gluonpolarization vector $e^{m}$ and the gluino wave function $\chi^{\alpha}$ and can be expanded in the Grassmann odd superspace coordinates $\theta^{\alpha}$.

They satisfy the following linearized equations of motion,

$$
\begin{gathered}
D_{\alpha} A_{\beta}+D_{\beta} A_{\alpha}=\gamma_{\alpha \beta}^{m} A_{m}, \quad D_{\alpha} A_{m}=\left(\gamma_{m} W\right)_{\alpha}+k_{m} A_{\alpha}, \\
D_{\alpha} \mathcal{F}_{m n}=2 k_{[m}\left(\gamma_{n]} W\right)_{\alpha}, \quad D_{\alpha} W^{\beta}=\frac{1}{4}\left(\gamma^{m n}\right)_{\alpha}{ }^{\beta} \mathcal{F}_{m n}
\end{gathered}
$$

which imply the on-shell constraints $k_{m} e^{m}=k_{m} \gamma_{\alpha \beta}^{m} \chi^{\beta}=0$ in components.

The scattering amplitudes of these massless states are obtained by computing the correlation function

$$
\mathcal{A}_{n}=\left\langle V^{1}(0) V^{(n-1)}(1) V^{n}(\infty) \int d z_{2} U^{2}\left(z_{2}\right) \ldots \int d z_{(n-2)} U^{(n-2)}\left(z_{(n-2)}\right)\right\rangle,
$$

\footnotetext{
1 For reviews of the pure spinor formalism, see $[10,11]$.
} 
where $V^{i}$ and $U^{i}$ are vertex operators writen in terms of the SYM superfields

$$
V^{i}=\lambda^{\alpha} A_{\alpha}^{i}(x, \theta), \quad U^{i}=\partial \theta^{\alpha} A_{\alpha}^{i}+\Pi^{m} A_{m}^{i}+d_{\alpha} W_{i}^{\alpha}+\frac{1}{2} \mathcal{F}_{m n}^{i} N^{m n} .
$$

The bosonic ghost field $\lambda^{\alpha}(z)$ is a pure spinor satisfying $\lambda^{\alpha} \gamma_{\alpha \beta}^{m} \lambda^{\beta}=0$ and

$$
\Pi^{m}(z)=\partial X^{m}+\frac{1}{2}\left(\theta \gamma^{m} \partial \theta\right), \quad d_{\alpha}(z)=\partial_{\alpha}-\frac{1}{2}\left(\gamma^{m} \theta\right)_{\alpha} \partial X_{m}-\frac{1}{8}\left(\gamma^{m} \theta\right)_{\alpha}\left(\theta \gamma_{m} \partial \theta\right)
$$

are, respectively, the supersymmetric momentum and Green-Schwarz constraint. The Lorentz currents for the pure spinor field are $N^{m n}(z)=\frac{1}{2}\left(\lambda \gamma^{m n} w\right)$, where $w_{\alpha}(z)$ is the conjugate momentum of $\lambda^{\alpha}(z)$ [9]. Their OPE's are easily computed [10,13].

The pure spinor BRST charge is defined by [9]

$$
Q=\oint \lambda^{\alpha}(z) d_{\alpha}(z)
$$

and satisfies $Q^{2}=0$. It can be shown that imposing $Q V=0$ puts all superfields on-shell, which also implies that the BRST variation of the integrated vertex $U(z)$ can be written as $Q U=\partial V[10]$ (see also [14]).

After using the OPE's to integrate out the conformal-weight one variables from the correlator (2.2), one is left with an expression containing only zero-modes of the form

$$
\mathcal{A}_{n}=\left\langle\lambda^{\alpha} \lambda^{\beta} \lambda^{\gamma} f_{\alpha \beta \gamma}^{i_{1} \ldots i_{n}}\left(\theta, \alpha^{\prime}\right)\right\rangle
$$

In (2.6), $f_{\alpha \beta \gamma}^{i_{1} \ldots i_{n}}\left(\theta, \alpha^{\prime}\right)$ is both a composite superfield in the labels $\left[i_{1}, \ldots i_{n}\right]$ of the external states and a function of the string scale $\alpha^{\prime}$ satisfying $\lambda^{\alpha} \lambda^{\beta} \lambda^{\gamma} \lambda^{\delta} D_{\delta} f_{\alpha \beta \gamma}^{i_{1} \ldots i_{n}}\left(\theta, \alpha^{\prime}\right)=0$ due t BRST invariance. Its specific form in terms of the super Yang-Mills superfields $\left[A_{\alpha}^{i}, A_{m}^{i}, W_{i}^{\alpha}, \mathcal{F}_{m n}^{i}\right]$ follows from OPE contractions while its functional dependence on $\alpha^{\prime}$ is determined by the momentum expansion of $n$-point Gaussian hypergeometric functions [15] and multiple Gaussian hypergeometric functions [16,17]. The zero-mode integration is denoted by the pure spinor bracket $\langle\ldots\rangle[9]$. It selects from the $\theta$-expansion $[18,19]$ of the enclosed superfields the unique element in the cohomology of the pure spinor BRST operator at ghost-number three, and its tree-level normalization can be chosen as

$$
\left\langle\left(\lambda \gamma^{m} \theta\right)\left(\lambda \gamma^{n} \theta\right)\left(\lambda \gamma^{p} \theta\right)\left(\theta \gamma_{m n p} \theta\right)\right\rangle=1 .
$$

Although (2.7) involves only five $\theta$ 's it can be shown to be supersymmetric [9]. Furthermore, given the fact that there is only one scalar in the decomposition of $\lambda^{3} \theta^{5}$, any $\left\langle\lambda^{\alpha} \lambda^{\beta} \lambda^{\gamma} f_{\alpha \beta \gamma}(\theta)\right\rangle$ can be determined using symmetry arguments alone. This zero-mode integration has been automated in a FORM [20] program [21] and therefore the component expansions of any supersymmetric amplitude computed with the pure spinor formalism are readily obtained. However, it is much more convenient to study the properties of the amplitudes directly in the superspace expressions, where the BRST cohomology properties of the pure spinor superspace allow several simplications to be carried out $[22,23]$. 


\subsection{BRST building blocks}

In [5], the cohomology nature of pure spinor superspace has been exploited to define natural objects $T_{i}, T_{i j}, T_{i j k}, T_{i j k l}, \ldots$ which transform covariantly under the BRST charge of (2.5). These so-called BRST building blocks $T_{i j k \ldots}$ are constructed from the OPE's among the vertex operators $V_{i}\left(z_{i}\right) U_{j}\left(z_{j}\right) U_{k}\left(z_{k}\right) \ldots$ and are defined in such a way as to not contain any BRST-exact terms [5]. They are ultimately written in terms of super YangMills superfields and therefore their $\theta$-expansions are also known; for example the building block with one label is given by $T_{i} \equiv V_{i}$ where $V_{i}$ is the vertex operator of (2.3). The next building block $T_{i j}$ is an antisymmetric combination

$$
T_{i j}=\frac{1}{2}\left(L_{j i}-L_{i j}\right)
$$

of the OPE residue $L_{j i}=-A_{m}^{i}\left(\lambda \gamma^{m} W^{j}\right)-V^{i}\left(k^{i} \cdot A^{j}\right)$ of $V_{i}\left(z_{i}\right) U_{j}\left(z_{j}\right)$. In [5], all building blocks up to and including $T_{i j k l m}$ have been explicitly expressed in terms of SYM superfields. As already mentioned, they transform covariantly under the BRST charge [5],

$$
\begin{aligned}
Q T_{12} & =s_{12} V_{1} V_{2}, \quad Q T_{123}=\left(s_{13}+s_{23}\right) T_{12} V_{3}+s_{12}\left(V_{1} T_{23}+T_{13} V_{2}\right) \\
Q T_{12 \ldots n} & =\sum_{j=2}^{n} \sum_{\alpha \in P\left(\beta_{j}\right)}\left(s_{1 j}+s_{2 j}+\ldots+s_{j-1, j}\right) T_{12 \ldots j-1,\{\alpha\}} T_{j,\left\{\beta_{j} \backslash \alpha\right\}},
\end{aligned}
$$

where $\beta_{j}=\{j+1, \ldots, n\}$ and $P\left(\beta_{j}\right)$ is the power set of $\beta_{j}$. The $s_{i j}=k_{i} \cdot k_{j}$ denote standard Mandelstam variables.

Furthermore, the BRST building blocks $T_{i_{1} i_{2} \ldots i_{p}}$ have several symmetry properties which leave $(p-1)$ ! independent permutations at rank $p$ and match the symmetries of the cubic diagrams they represent, e.g.

$$
0=T_{12}+T_{21}=T_{123}+T_{213}=T_{123}+T_{231}+T_{312}
$$

The rank $p$ object $T_{i_{1} i_{2} \ldots i_{p}}$ inherits all symmetries of its lower rank relative $T_{i_{1} i_{2} \ldots i_{p-1}}$ in the first $p-1$ labels. It is therefore sufficient to give the novel relation at each rank which involves all the $p$ indices $^{2}$,

$$
\begin{array}{cl}
p=2 n+1: & T_{12 \ldots n+1[n+2[\ldots[2 n-1[2 n, 2 n+1]] \ldots]]}-2 T_{2 n+1 \ldots n+2[n+1[\ldots[3[21]] \ldots]]}=0 \\
p=2 n: & T_{12 \ldots n[n+1[\ldots[2 n-2[2 n-1,2 n]] \ldots]]}+T_{2 n \ldots n+1[n[\ldots[3[21]] \ldots]]}=0,
\end{array}
$$

2 The notation $[i[j k]]$ using nested square brackets means consecutive antisymmetrization of pairs of labels starting from the outmost one, e.g. $T_{[i[j k]]}=1 / 2\left(T_{i[j k]}-T_{[j k] i}\right)=1 / 4\left(T_{i j k}-T_{i k j}-\right.$ $\left.T_{j k i}+T_{k j i}\right)$ 


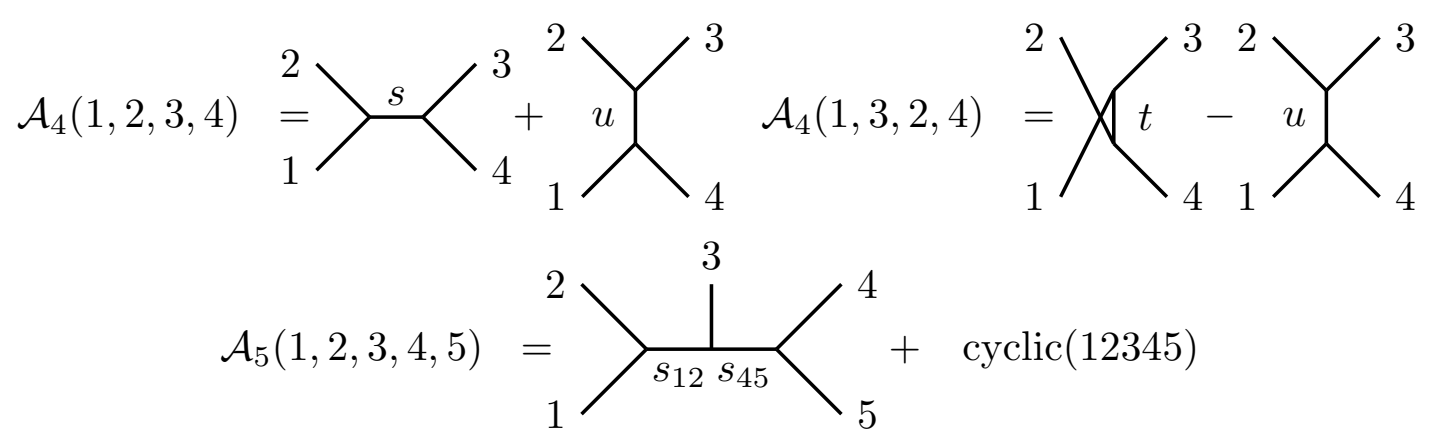

Fig. 1 The four- and five-point amplitudes in terms of cubic graphs. The other five-point diagrams for the Kleiss-Kuijf basis can be obtained by relabeling.

e.g. $T_{1234}+T_{1243}+T_{4321}+T_{4312}=0$ at rank four.

Making use of these building blocks we have obtained the general form of the tree-level $n$ point correlator of $(2.2)$ in [5,24] (see equation (3.5) below). As will now be discussed, the field-theory limit of the superstring amplitude can be used to find supersymmetric and local $n$-point BCJ-satisfying kinematic numerators [1] in a straightforward manner.

\section{String inspired BCJ numerators in superspace}

According to the hypothesis of BCJ, the color dressed $n$ point tree amplitude in gauge theories can be parametrized as

$$
\mathcal{A}_{n}=\sum_{i} \frac{c_{i} n_{i}}{\prod_{\alpha_{i}} s_{\alpha_{i}}}
$$

such that the kinematic factors $n_{i}$ satisfy Jacobi-like relations in one-to-one correspondence with the group-theoretic Jacobi identities for the color factors $c_{i}$,

$$
c_{i} \pm c_{j} \pm c_{k}=0 \quad \Rightarrow \quad n_{i} \pm n_{j} \pm n_{k}=0 .
$$

The relative signs depend on the choice of signs when defining the color factors. The $i$ sum in (3.1) runs over the $(2 n-5)$ !! cubic diagrams or pole channels specified by $n-3$ propagators $s_{\alpha_{i}}^{-1}$.

In this section, we will derive the kinematic numerators $n_{i}$ in this organization scheme from superstring theory. Basic counting arguments together with relation between color ordered amplitudes imply that these $n_{i}$ satisfy the Jacobi identities (3.2) by construction. 


\subsection{The field theory setup}

The set of $(n-2)$ ! color ordered amplitudes $\mathcal{A}_{n}\left(1,2_{\rho}, \ldots,(n-1)_{\rho}, n\right)$ with $\rho \in S_{n-2}$ and legs $n$ and 1 fixed is sufficient to involve all of the $(2 n-5)$ !! diagrams at least once. According to the Kleiss-Kuijf relations [25] all the other subamplitudes are sums over several $\mathcal{A}_{n}\left(1,2_{\rho}, \ldots,(n-1)_{\rho}, n\right)$ with coefficients \pm 1 . That is why we will refer to them as the KK basis in the following. The $(n-2)$ ! amplitudes at four-points are given by

$$
\mathcal{A}_{4}(1,2,3,4)=\frac{n_{s}}{s}+\frac{n_{u}}{u}, \quad \mathcal{A}_{4}(1,3,2,4)=\frac{n_{t}}{t}-\frac{n_{u}}{u}
$$

while for five-points [1],

$$
\begin{aligned}
& \mathcal{A}_{5}(1,2,3,4,5)=\frac{n_{1}}{s_{12} s_{45}}+\frac{n_{2}}{s_{23} s_{51}}+\frac{n_{3}}{s_{12} s_{34}}+\frac{n_{4}}{s_{23} s_{45}}+\frac{n_{5}}{s_{34} s_{51}} \\
& \mathcal{A}_{5}(1,4,3,2,5)=\frac{n_{6}}{s_{14} s_{25}}+\frac{n_{5}}{s_{34} s_{51}}+\frac{n_{7}}{s_{23} s_{14}}+\frac{n_{8}}{s_{34} s_{25}}+\frac{n_{2}}{s_{23} s_{51}} \\
& \mathcal{A}_{5}(1,3,4,2,5)=\frac{n_{9}}{s_{13} s_{25}}-\frac{n_{5}}{s_{34} s_{51}}+\frac{n_{10}}{s_{13} s_{24}}-\frac{n_{8}}{s_{34} s_{25}}+\frac{n_{11}}{s_{51} s_{24}} \\
& \mathcal{A}_{5}(1,2,4,3,5)=\frac{n_{12}}{s_{12} s_{35}}+\frac{n_{11}}{s_{24} s_{51}}-\frac{n_{3}}{s_{12} s_{34}}+\frac{n_{13}}{s_{35} s_{24}}-\frac{n_{5}}{s_{51} s_{34}} \\
& \mathcal{A}_{5}(1,4,2,3,5)=\frac{n_{14}}{s_{14} s_{35}}-\frac{n_{11}}{s_{24} s_{51}}-\frac{n_{7}}{s_{14} s_{23}}-\frac{n_{13}}{s_{24} s_{35}}-\frac{n_{2}}{s_{23} s_{51}} \\
& \mathcal{A}_{5}(1,3,2,4,5)=\frac{n_{15}}{s_{13} s_{45}}-\frac{n_{2}}{s_{23} s_{51}}-\frac{n_{10}}{s_{13} s_{24}}-\frac{n_{4}}{s_{23} s_{45}}-\frac{n_{11}}{s_{24} s_{51}}
\end{aligned}
$$

and their representation in terms of cubic graphs is depicted in Fig. 1. One possible parametrization for the six point amplitude can be found in [26].

As mentioned in the introduction, the $n_{i}$ are not uniquely specified by the parametrization of $\mathcal{A}_{4}$ and $\mathcal{A}_{5}$ shown above. There still is a freedom to add zeros of the form

$0=\left(\frac{s_{i j}}{s_{i j}}-\frac{s_{k l}}{s_{k l}}\right) \times(\ldots)$ to individual subamplitudes which amounts to reabsorbing contact terms into a different numerator $n_{i}$.

At four point level, the only Jacobi-like identity $n_{s}+n_{t}-n_{u}=0$ holds independent of the ambiguity of reshuffling contact terms between the numerators. This is a feature of the simple structure of $\mathcal{A}_{4}$ and its momentum phase space.

In $\mathcal{A}_{5}$, imposing the color algebra on the fifteen $n_{i}$ yields nine independent relations

$$
\begin{aligned}
& 0=n_{3}-n_{5}+n_{8}=n_{3}-n_{1}+n_{12}=n_{10}-n_{11}+n_{13} \\
& 0=n_{4}-n_{2}+n_{7}=n_{4}-n_{1}+n_{15}=n_{10}-n_{9}+n_{15} \\
& 0=n_{8}-n_{6}+n_{9}=n_{5}-n_{2}+n_{11}=n_{7}-n_{6}+n_{14}
\end{aligned}
$$


which leave six independent numerators. However, the kinematic Jacobi identities at five and higher points generically fail to hold unless a specific class of choices for the $n_{i}$ is made.
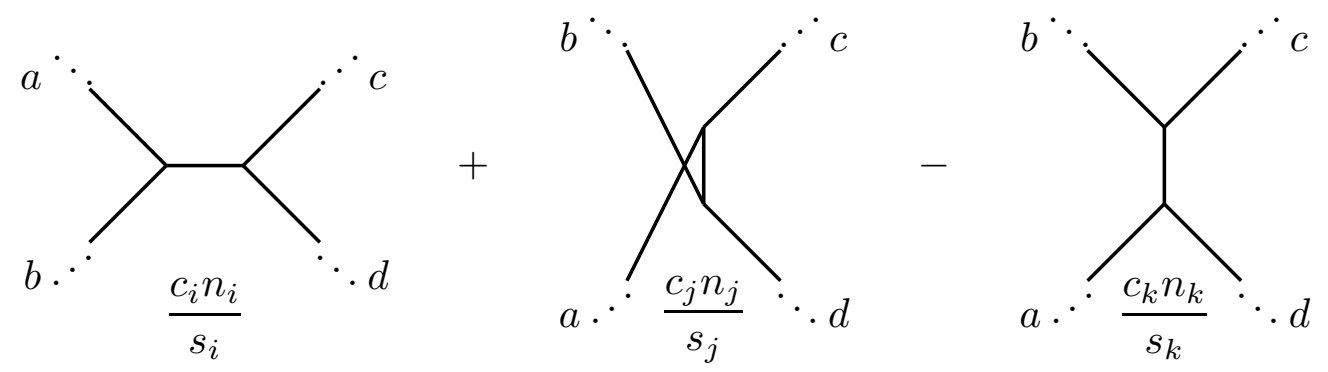

Fig. 2 Triplet of subdiagrams where the sum over the associated color factor vanishes due to the Jacobi identity $f^{e[a b} f^{c] d e}=0$.

More generally, if a suitable parametrization and contact term bookkeeping is chosen the duality between color and kinematics manifests itself for each triplet of subdiagrams shown in figure Fig. 2. For cubic graphs describing an $n$-point tree amplitude, there can be arbitrary further subdiagrams $a, b, c, d$ attached to the dotted lines with $n-4$ common propagators in total.

In an $n$-point tree level amplitude, if all the Jacobi-like identities for the $(2 n-5)$ !! numerators in $\mathcal{A}_{n}$ are satisfied, then a set of $(n-2)$ ! independent $n_{i}$ remains. In the following we will reverse the line of reasoning: If one can show that the $\mathrm{KK}$ basis $\mathcal{A}_{n}\left(1,2_{\sigma}, \ldots,(n-1)_{\sigma}, n\right)$ of color ordered amplitudes can be expressed in terms of $(n-2)$ ! basis numerators, then there must be as many equations between the larger set of $(2 n-5) ! !$ numerators as there are Jacobi identities.

\subsection{A minimal kinematic basis from superstring theory}

Supersymmetric field theory tree amplitudes can also be obtained from the low-energy limit of superstring theory where the dimensionless combinations $\alpha^{\prime} s_{i_{1} \ldots i_{p}}$ of Regge slope $\alpha^{\prime}$ and Mandelstam bilinears $s_{i_{1} \ldots i_{p}}=\frac{1}{2}\left(k_{i_{1}}+k_{i_{2}}+\ldots+k_{i_{p}}\right)^{2}$ are formally sent to zero. Using the pure spinor formalism [9], we will show in [5] that the color stripped superstring $n$-point amplitude is given by

$$
\begin{aligned}
& \mathcal{A}_{n}^{\text {string }}\left(1_{\sigma}, 2_{\sigma}, \ldots,(n-1)_{\sigma}, n ; \alpha^{\prime}\right)=\left(2 \alpha^{\prime}\right)^{n-3} \prod_{i=2}^{n-2} \int_{\mathcal{I}_{\sigma}} \mathrm{d} z_{i} \prod_{j<k}\left|z_{j k}\right|^{-2 \alpha^{\prime} s_{j k}} \\
& \sum_{j=1}^{n-2} \frac{\left\langle T_{12 \ldots j} T_{n-1, n-2 \ldots j+1} V_{n}\right\rangle}{\left(z_{12} z_{23} \ldots z_{p-1, p}\right)\left(z_{n-1, n-2} z_{n-2, n-3} \ldots z_{j+2, j+1}\right)}+\mathcal{P}(2,3, \ldots, n-2)
\end{aligned}
$$


in terms of the BRST building blocks $T_{12 \ldots p}$, with $z_{j k}=z_{j}-z_{k}$. The $\mathcal{P}(2,3, \ldots, n-2)$ denotes the sum over all permutations of the labels $2,3, \ldots, n-2$ of the integrated vertex operators (2.3).

The ordering $\sigma \in S_{n-1}$ of the external legs is reflected in the integration region $\mathcal{I}_{\sigma}$ for the worldsheet positions $z_{2_{\sigma}}, z_{3_{\sigma}}, \ldots, z_{(n-2)_{\sigma}}$, the remaining ones are fixed as $\left(z_{1_{\sigma}}, z_{(n-1)_{\sigma}}, z_{n}\right)=(0,1, \infty)$ by $S L(2, R)$ invariance of the disk worldsheet. More precisely, $\mathcal{I}_{\sigma}$ is defined such that only those $z_{i}$ which respect the ordering

$$
0=z_{1_{\sigma}} \leq z_{2_{\sigma}} \leq z_{3_{\sigma}} \leq \ldots \leq z_{(n-2)_{\sigma}} \leq z_{(n-1)_{\sigma}}=1
$$

are integrated over.

To arrive at (3.5) from the pure spinor conformal field theory (CFT), one has to reexpress integrals with double pole factors like $z_{i j}^{-2}$ in terms of single pole integrals (with integrands such as $\left.\left(z_{i j} z_{j k}\right)^{-1}\right)$. The superfields associated with these world-sheet poles conspire such that the OPE residues of $V_{1}\left(z_{1}\right) U_{2}\left(z_{2}\right) \ldots U_{p}\left(z_{p}\right)$ in single pole integrals receive all the corrections necessary to form BRST building blocks $T_{12 \ldots p}$. It has already been realized in $[6,7]$ that double poles cause various technical complications and in particular prevent the basis of kinematics to boil down to the desirable size of $(n-2)$ !.

The $\alpha^{\prime} \rightarrow 0$ limit of (3.5) extracts propagators of cubic field theory diagrams from the $n-3$ fold worldsheet integrals. Adjusting the integration region $\mathcal{I}_{\sigma}$ to the color ordering makes sure that the integrals in $\mathcal{A}_{n}^{\text {string }}\left(1_{\sigma}, 2_{\sigma}, \ldots,(n-1)_{\sigma}, n ; \alpha^{\prime}\right)$ only generate those pole channels which appear in the corresponding field theory amplitude $\mathcal{A}_{n}\left(1_{\sigma}, 2_{\sigma}, \ldots,(n-\right.$ $\left.1)_{\sigma}, n\right)$. A method to efficiently extract the field theory limit of a general $n$-point integral is described in [5].

The remarkable property of (3.5) in view of the BCJ organization is the number of independent superfield kinematics $\left\langle T_{12 \ldots j} T_{n-1, n-2, \ldots, j+1} V_{n}\right\rangle$. Each of the $n-2$ terms in the $j$ sum of (3.5) involves $(n-3)$ ! permutations of $\left\langle T_{12 \ldots j} T_{n-1, n-2, \ldots, j+1} V_{n}\right\rangle$ in the legs $(2,3, \ldots, n-2)$ such that we have $(n-2)$ ! kinematic packages in total. The world-sheet integrand remains the same for any color ordering, only the integration region $\mathcal{I}_{\sigma}$ changes between the subamplitudes.

Hence, the $(n-2)$ ! basic kinematics $\left\langle T_{12 \ldots j} T_{n-1, n-2, \ldots, j+1} V_{n}\right\rangle$ combine to the kinematic factors for any color stripped superstring amplitude, and in particular, they generate all the $(2 n-5)$ !! BCJ numerators of the field theory amplitude in the $\alpha^{\prime} \rightarrow 0$ limit. Their coefficients are determined by the pole structure of the integrals in the corresponding 
integration region $\mathcal{I}_{\sigma}$ which is specified by the color ordering of $\mathcal{A}_{n}\left(1,2_{\sigma}, 3_{\sigma}, \ldots,(n-\right.$ 1) $\sigma, n)$.

As we have argued in the previous subsection - having a set of no more than $(n-2)$ ! independent numerators is necessary for imposing the Jacobi-like identities (dual to color factors) on the $(2 n-5)$ !! numerators of the pole channels in various subamplitudes. In the next paragraph we explain why the number $(n-2)$ ! of kinematics in $(3.5)$ is also sufficient to satisfy the Jacobi relations.

\subsection{The vanishing of numerator triplets}

The fact that only $(n-2)$ ! BCJ numerators can be linearly independent implies the existence of as many linear homogeneous relations between the $n_{i}$ as there are Jacobi identities. Since the field theory limits of the integrals in (3.5) involve no other coefficients than 0 and \pm 1 for the propagators, these relations must be of the form

$$
n_{i_{1}} \pm n_{i_{2}} \mp n_{i_{3}} \pm \ldots \mp n_{i_{p-1}} \pm n_{i_{p}}=0
$$

with a so far unspecified number $p$ of terms. In order to show that they can always be arranged into vanishing statements for triplets $n_{i_{1}} \pm n_{i_{2}} \mp n_{i_{3}}=0$ one has to make use of the monodromy relations of field theory [1]

$$
s_{12} \mathcal{A}_{n}(2,1,3, \ldots, n)+\sum_{j=3}^{n-1}\left(\sum_{k=2}^{j} s_{1 k}\right) \mathcal{A}_{n}(2,3, \ldots, j, 1, j+1, \ldots, n-1, n)=0
$$

which allow to reduce the KK subamplitudes to a basis of $(n-3)$ ! independent ones. Their string theory generalization replaces the sum $\sum_{k} s_{1 k}$ by sine functions $\sin \left(2 \alpha^{\prime} \pi \sum_{k} s_{1 k}\right)$ $[27,28]$.

By taking appropriate permutations of (3.7) and decomposing the occurring subamplitudes in pole channels, one can derive identities between Jacobi triplets $\left(n_{i_{k}}, n_{i_{l}}, n_{i_{m}}\right)$ dual to color factors with $c_{i_{k}}+c_{i_{l}}+c_{i_{m}}=0$ of the following form $[7,29]$

$$
\sum_{i} \frac{n_{i_{k}}+n_{i_{l}}+n_{i_{m}}}{\prod_{\alpha_{i}}^{n-4} s_{\alpha_{i}}}=0
$$

The $i$ sum runs over $n-1$ point channels of total number $2^{n-3}(2 n-7) ! !(n-3) /(n-2)$ ! and involves the $n-4$ propagators $s_{\alpha_{i}}$ common among the $n_{i_{k}}, n_{i_{l}}$ and $n_{i_{m}}$ channels. As a consquence, $n_{i_{k}}+n_{i_{l}}+n_{i_{m}}$ must vanish at the residue of the $n-4$ poles, independent on the assignment of contact terms to the numerators. 
Suppose the linear dependences (3.6) failed to make Jacobi triplets of BCJ numerators vanish, i.e. $p>3$, then (3.8) would involve terms

$$
\frac{n_{i_{1}}+n_{i_{2}}+n_{i_{3}}}{\prod_{\alpha_{i}}^{n-4} s_{\alpha_{i}}}=-\frac{\sum_{j=4}^{p} n_{i_{j}}}{\prod_{\alpha_{i}}^{n-4} s_{\alpha_{i}}}
$$

where each noncancelling $n_{i_{j>3}}$ is muliplied with at least one propagator outside its channels. This is clearly incompatible with (3.8) because there will remain contributions with a specific set of $n-4$ poles from each term like that ${ }^{3}$.

The conclusion is that the $(2 n-5) ! !-(n-2)$ ! relations $(3.6)$ can always be brought into a form that reproduces all the dual Jacobi identities for the kinematic numerators $n_{i}$. If this was not the case, inconsistencies would arise in the monodromy relations (3.7) or (3.8) between color ordered field theory amplitudes. Hence, the number $(n-2)$ ! of kinematics in (3.5) guarantees that all the $n_{i}$ constructed from the $\alpha^{\prime} \rightarrow 0$ limit of the integrals over $\mathcal{I}_{\sigma}$ satisfy the Jacobi-like relations $n_{i}+n_{j}+n_{k}=0$.

\subsection{The explicit formula for BCJ numerators}

The worldsheet integrand of (3.5) suggests to label the $(n-2)$ ! basis kinematics in an $n$-point amplitude by an $S_{n-3}$ permutation $\sigma$ and an integer $l=1, \ldots, n-2$ :

$$
\mathcal{K}_{\sigma}^{l}=\left\langle T_{12_{\sigma} 3_{\sigma} \ldots l_{\sigma}} T_{n-1,(n-2)_{\sigma} \ldots(l+1)_{\sigma}} V_{n}\right\rangle, \quad l=1, \ldots, n-2, \quad \sigma \in S_{n-3}
$$

This makes sure that the residual $S_{n-3}$ relabelling symmetry stays visible in the KK basis of the field theory limit. As we have mentioned before, knowing all the KK subamplitudes $\mathcal{A}_{n}\left(1,2_{\rho}, \ldots,(n-1)_{\rho}, n\right)$ is sufficient to address each channel and to thereby identify all the $(2 n-5)$ !! numerators $n_{i}$. The superstring amplitude $(3.5)$ provides a general prescription to construct these KK subamplitudes in terms of the basis kinematics $\mathcal{K}_{\sigma}^{l}$ defined by (3.9)

$$
\mathcal{A}_{n}\left(1,2_{\rho}, \ldots,(n-1)_{\rho}, n\right)=\sum_{l=1}^{n-2} \sum_{\sigma \in S_{n-3}} P_{\rho}^{(l, \sigma)} \mathcal{K}_{\sigma}^{l}
$$

3 The claimed incompatibility rests on the linear independence of the $(n-2)$ ! factorial basis numerators. We wish to thank Henrik Johansson for pointing out that a loophole would arise otherwise. 
This introduces a $(n-2) ! \times(n-2)$ ! matrix $P_{\rho}^{(l, \sigma)}$ of kinematic poles whose entries is determined by the integral of the worldsheet polynomial associated with $(l, \sigma)$ over the integration region $\mathcal{I}_{\rho}$ :

$$
P_{\rho}{ }^{(l, \sigma)}=\lim _{\alpha^{\prime} \rightarrow 0}\left(2 \alpha^{\prime}\right)^{n-3} \prod_{i=2}^{n-2} \int_{\mathcal{I}_{\rho}} \mathrm{d} z_{i} \frac{\prod_{j<k}\left|z_{j k}\right|^{-2 \alpha^{\prime} s_{j k}}}{z_{12_{\sigma}} z_{2_{\sigma} 3_{\sigma}} \ldots z_{(l-1)_{\sigma} l_{\sigma}} z_{n-1,(n-2)_{\sigma}} \ldots z_{(l+2)_{\sigma},(l+1)_{\sigma}}}
$$

These $\alpha^{\prime} \rightarrow 0$ limits can be straightforwardly evaluated using the methods of [5].

The idea of introducing an $(n-2)$ ! vector of KK amplitudes and relating it to $(n-2)$ ! independent numerators by a square matrix already appeared in [30]. In our situation, the basis (3.9) of numerators is set by the superstring computation, and our $P_{\rho}^{(l, \sigma)}$ matrix is a specialization of the propagator matrix $M$ in this reference to the pure spinor basis of kinematics. The linear dependences of KK subamplitudes due to BCJ relations (3.7) imply that the $(n-2) ! \times(n-2)$ ! propagator matrices $M$ or $P_{\rho}^{(l, \sigma)}$ have rank $(n-3) !$.

Not all the entries of the pole matrix $P_{\rho}{ }^{(l, \sigma)}$ have to be computed separately. The following trick relates many of them by relabelling and thereby reduces the computational effort on the way towards explicit BCJ numerators: Exclude the leg $n-1$ from the $\rho \in$ $S_{n-2}$ permutations specifying the KK subamplitudes. They then fall into $n-2$ classes $\mathcal{A}_{n}\left(1,2_{\sigma}, \ldots, j_{\sigma}, n-1,(j+1)_{\sigma}, \ldots,(n-2)_{\sigma}, n\right)$ with $j-1$ legs between 1 and $n-1$ and another $n-2-j$ legs between $n-1$ and $n$. The legs $2,3, \ldots, n-2$ are interchanged by $S_{n-3}$ permutations $\sigma$ like in (4.4). It is sufficient to compute one representative of the $n-2$ classes of KK amplitudes, the others then follow as $S_{n-3}$ permutations in $2,3, \ldots, n-2$. More precisely, once the first $n-2$ columns of $(3.10)$ with $\rho=(2,3, \ldots, j, n-1, j+1, \ldots, n-2)$ and $j=1,2, \ldots, n-2$ are known, then the others follow from

$$
P_{\rho=\left(2_{\sigma}, 3_{\sigma}, \ldots, j_{\sigma}, n-1,(j+1)_{\sigma}, \ldots,(n-2)_{\sigma}\right)}^{(l, \tau)}=\left.P_{\rho=(2,3, \ldots, j, n-1, j+1, \ldots, n-2)}^{\left(l, \sigma^{-1} \circ \tau\right)}\right|_{k_{i} \mapsto k_{\sigma(i)}}
$$

where the concatenation of $\sigma^{-1}, \tau \in S_{n-3}$ is to be understood as $\left(\sigma^{-1} \circ \tau\right)(i)=\sigma^{-1}(\tau(i))$. The proof of (3.11) is a simple matter of bookkeeping with world-sheet integration variables in $(3.10)$.

This relabelling strategy reduces the number of independent evaluations of (3.10) from $(n-2) ! \times(n-2)$ ! down to $(n-2) \times(n-2)$ !, i.e. the work at this step is reduced by a factor of $(n-3)$ !. But the success of these $S_{n-3}$ relabellings does not extend to the leg $n-1$. The $P_{\rho}^{(l, \sigma)}$ entries for the $n-2$ classes of KK subamplitudes $\mathcal{A}_{n}\left(1,2_{\sigma}, \ldots, j_{\sigma}, n-\right.$ $\left.1,(j+1)_{\sigma}, \ldots,(n-2)_{\sigma}, n\right)$ with $j=1,2, \ldots, n-2$ have a different number and structure 
of terms as $j$ varies. This will become more obvious from the examples in the next section. As a consequence, the $n_{i}$ appearing in these subamplitudes involve more basic kinematics $\mathcal{K}_{\sigma}^{l}$ and do not follow from other BCJ numerators by relabelling.

\subsection{Jacobi-friendly notation}

At higher points it is not convenient to denote the BCJ numerators sequentially by $n_{i}$ for $i=1,2, \ldots(2 n-5)$ !!. Already the presentation of the fifteen numerators in the five point KK basis (3.3) suffers from the arbitrary assignment of numbers 1 to 15 to the cubic diagrams. It is not at all obvious from their labels which of them combine to form the Jacobi triplets (3.4).

Instead, we will use a notation introduced by [30] which reflects the structure of the diagram and allows the associated propagators to be reconstructed. More importantly, it makes the dual structure constant contraction available from which one can infer the symmetry properties $f^{a b c}=-f^{b a c}$ and the Jacobi identities $f^{b\left[a_{1} a_{2}\right.} f^{\left.a_{3}\right] b c}=0$. Let us clarify these properties by explicit examples:

The four points amplitude encompasses three diagrams of the form

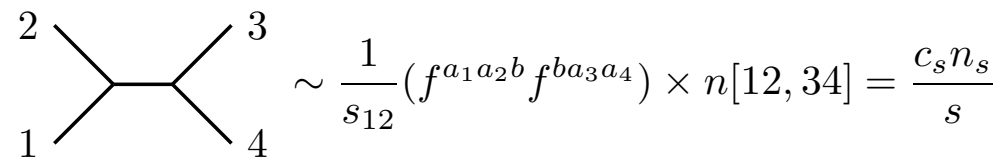

where $n[12,34]$ has the same symmetries as the structure constants involved:

$$
n[i j, k l]=-n[j i, k l]=-n[i j, l k]=n[j i, l k], \quad n[i j, k l]=n[k l, i j]
$$

If we assign $n_{t}=n[13,42]$ and $n_{u}=n[23,41]$, then the Jacobi identity $n_{s}+n_{t}-n_{u}=0$ can be written more compactly as

$$
n[1\{2,34\}]:=n[12,34]+n[13,42]+n[14,23]=0 .
$$

At five points, the first out of fifteen pole channels contributes

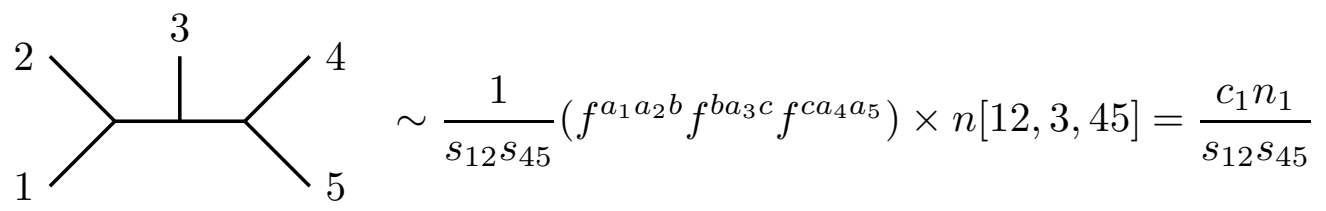

where the kinematic numerators inherit their antisymmetry under flipping a cubic vertex from the structure constants:

$$
n[i j, k, l m]=-n[j i, k, l m]=-n[i j, k, m l]=n[j i, k, m l], \quad n[i j, k, l m]=-n[l m, k, i j]
$$


All the Jacobi identities (3.4) can be diagrammatically found by attaching a cubic vertex with two external legs to one of the dotted lines of figure Fig. 2. They can be cast into unified form

$$
n[i j,\{k, l m\}]=0 .
$$

Six point amplitudes introduce two topologies of cubic diagrams

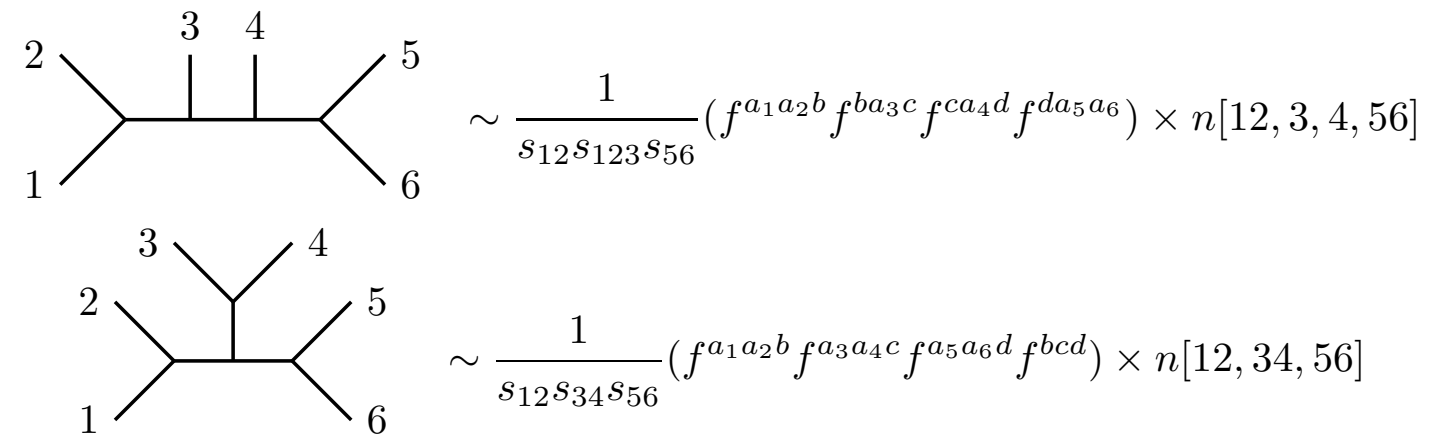

which imprint the following symmetries on the BCJ numerators:

$$
\begin{aligned}
n[i j, k, l, m p] & =-n[j i, k, l, m p], \quad n[i j, k, l, m p]=n[m p, l, k, i j] \\
n[i j, k l, m p] & =-n[j i, k l, m p], \quad n[i j, k l, m p]=-n[k l, i j, m p]
\end{aligned}
$$

Also the Jacobi identites exhibit different topologies here, one can either attach three point vertices to two different external lines of Fig. 2 or one color ordered four point diagram to one external line:

$$
n[i j, k,\{l, m p\}]=0, \quad n[i j, k l, m p]=n[i j, k, l, m p]-n[i j, l, k, m p]
$$

The latter expresses any diagram of snowflake shape in terms of the other topology.

Seven points again introduce two topologies of cubic diagrams

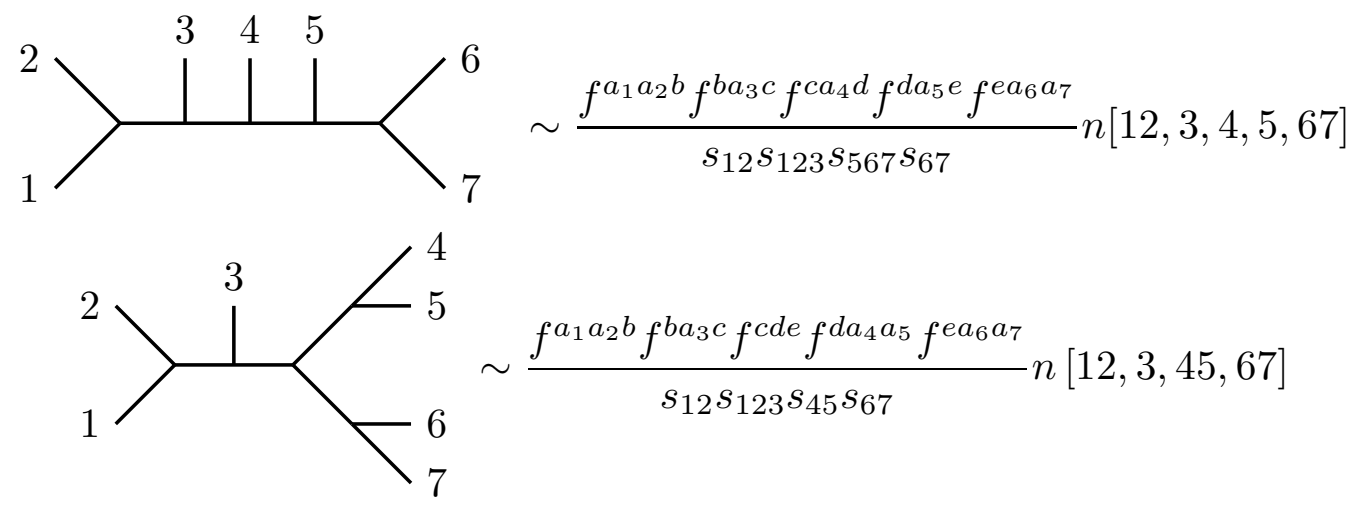

They give rise to symmetry properties

$$
\begin{gathered}
n[i j, k, l, m, p q]=-n[j i, k, l, m, p q], \quad n[i j, k, l, m, p q]=-n[p q, m, l, k, i j] \\
n[i j, k, l m, p q]=-n[j i, k, l m, p q]=-n[i j, k, m l, p q], \quad n[i j, k, l m, p q]=-n[i j, k, p q, l m]
\end{gathered}
$$


and Jacobi identities which eliminate the topology with more branchings:

$$
n[i j, k, l,\{m, p q\}]=0, \quad n[i j, k, l m, p q]=n[i j, k, l, m, p q]-n[i j, k, m, l, p q]
$$

The four different topologies at eight points can still be captured by the suggestive notations $n[i j, k, l, m, p, q r], n[i j, k, l m, p, q r], n[i j, k, l, m p, q r]$ and $n[k l, i j, m p, q r]$. Jacobi identities relate diagrams of different topology such that all of them can be represented in terms of the simplest numerators $n[i j, k, l, m, p, q r]$.

\section{Explicit examples}

In order to make the very general statements more tractable, we shall analyze explicit examples up to seven-point in detail.

\subsection{Four-point}

Let us first of all compute the four-point numerators as a warm-up exercise. For $n=4$, the general formula (3.5) gives rise to one worldsheet integral and two $z_{2}$ functions in the integrand,

$$
\mathcal{A}_{4}^{\text {string }}\left(1,2_{\rho}, 3_{\rho}, 4 ; \alpha^{\prime}\right)=2 \alpha^{\prime} \int_{\mathcal{I}_{\rho}} \mathrm{d} z_{2}\left|z_{2}\right|^{-2 \alpha^{\prime} s}\left|1-z_{2}\right|^{-2 \alpha^{\prime} u}\left\{\frac{\left\langle T_{12} V_{3} V_{4}\right\rangle}{z_{2}}+\frac{\left\langle V_{1} T_{23} V_{4}\right\rangle}{1-z_{2}}\right\}
$$

which can be easily evaluated in terms of the Euler Beta function. We obtain the following entries for the propagator matrix (3.10) by taking the field theory limit:

$$
\begin{aligned}
& \left(P_{(2,3)}{ }^{1}, P_{(2,3)}^{2}\right)=\lim _{\alpha^{\prime} \rightarrow 0} 2 \alpha^{\prime} \int_{0}^{1} \mathrm{~d} z_{2}\left|z_{2}\right|^{-2 \alpha^{\prime} s}\left|1-z_{2}\right|^{-2 \alpha^{\prime} u}\left(\frac{1}{z_{2}}, \frac{1}{1-z_{2}}\right)=\left(\frac{1}{s}, \frac{1}{u}\right) \\
& \left(P_{(3,2)}{ }^{1}, P_{(3,2)}{ }^{2}\right)=\lim _{\alpha^{\prime} \rightarrow 0} 2 \alpha^{\prime} \int_{1}^{\infty} \mathrm{d} z_{2}\left|z_{2}\right|^{-2 \alpha^{\prime} s}\left|1-z_{2}\right|^{-2 \alpha^{\prime} u}\left(\frac{1}{z_{2}}, \frac{1}{1-z_{2}}\right)=\left(\frac{1}{t},-\frac{1}{u}-\frac{1}{t}\right)
\end{aligned}
$$

The $S_{n-3}$ permutation $\sigma$ which appears as a superscript label of the general $P_{\rho}{ }^{(l, \sigma)}$ becomes trivial at four points. This leads to field theory subamplitudes

$$
\mathcal{A}_{4}(1,2,3,4)=\frac{n_{s}}{s}+\frac{n_{u}}{u}, \quad \mathcal{A}_{4}(1,3,2,4)=\frac{n_{s}-n_{u}}{t}-\frac{n_{u}}{u}=-\frac{n_{t}}{t}-\frac{n_{u}}{u}
$$

with BCJ numerators that manifestly satisfy a Jacobi relation:

$$
n_{s}=\left\langle T_{12} V_{3} V_{4}\right\rangle, \quad n_{u}=\left\langle V_{1} T_{23} V_{4}\right\rangle, \quad n_{t}=n_{u}-n_{s}=\left\langle V_{1} T_{23} V_{4}\right\rangle-\left\langle T_{12} V_{3} V_{4}\right\rangle
$$

They are evaluated in superfield components in appendix C. 


\subsection{Five-point}

The color ordered five-point superstring amplitude encompasses six basis kinematics:

$$
\begin{gathered}
\mathcal{A}_{5}^{\text {string }}\left(1,2_{\rho}, 3_{\rho}, 4 \rho, 5 ; \alpha^{\prime}\right)=\left(2 \alpha^{\prime}\right)^{2} \int_{\mathcal{I}_{\rho}} \mathrm{d} z_{2} \mathrm{~d} z_{3} \prod_{j<k}\left|z_{j k}\right|^{-2 \alpha^{\prime} s_{j k}} \\
\left(\frac{\left\langle T_{123} V_{4} V_{5}\right\rangle}{z_{12} z_{23}}+\frac{\left\langle T_{132} V_{4} V_{5}\right\rangle}{z_{13} z_{32}}+\frac{\left\langle T_{12} T_{43} V_{5}\right\rangle}{z_{12} z_{43}}+\frac{\left\langle T_{13} T_{42} V_{5}\right\rangle}{z_{13} z_{42}}+\frac{\left\langle V_{1} T_{432} V_{5}\right\rangle}{z_{43} z_{32}}+\frac{\left\langle V_{1} T_{423} V_{5}\right\rangle}{z_{42} z_{23}}\right)
\end{gathered}
$$

The double pole $z_{23}^{-2}$ appearing in the five point integrand of other references [6,7] was absorbed into the single-pole terms such that BRST building blocks $T_{i j}$ and $T_{i j k}$ could be formed. Absence of double poles is crucial for satisfying all the dual Jacobi relations and its removal was essentially dictated by the BRST cohomology properties of those building blocks $[5,26]$.

Let us rewrite the superfields in the $\mathcal{K}_{\sigma}^{l}$ notation of subsection 3.4 in order to make better use of the residual relabelling symmetry in $2 \leftrightarrow 3$ :

$$
\begin{aligned}
& \mathcal{K}_{(23)}^{3}=\left\langle T_{123} V_{4} V_{5}\right\rangle, \quad \mathcal{K}_{(23)}^{2}=\left\langle T_{12} T_{43} V_{5}\right\rangle, \quad \mathcal{K}_{(23)}^{1}=\left\langle V_{1} T_{432} V_{5}\right\rangle \\
& \mathcal{K}_{(32)}^{3}=\left\langle T_{132} V_{4} V_{5}\right\rangle, \quad \mathcal{K}_{(32)}^{2}=\left\langle T_{13} T_{42} V_{5}\right\rangle, \quad \mathcal{K}_{(32)}^{1}=\left\langle V_{1} T_{423} V_{5}\right\rangle
\end{aligned}
$$

Performing the field theory limit of the integrals (4.2) gives rise to the following six KK subamplitudes (where the permutation $\sigma$ of 2 and 3 can be kept general because of (3.11))

$$
\begin{aligned}
& \mathcal{A}_{5}\left(1,2_{\sigma}, 3_{\sigma}, 4,5\right)=\frac{\mathcal{K}_{\sigma(23)}^{3}}{s_{45} s_{12_{\sigma}}}+\frac{\mathcal{K}_{\sigma(23)}^{1}-\mathcal{K}_{\sigma(32)}^{1}}{s_{51} s_{2_{\sigma} 3_{\sigma}}}-\frac{\mathcal{K}_{\sigma(23)}^{2}}{s_{12_{\sigma}} s_{3_{\sigma}}}+\frac{\mathcal{K}_{\sigma(23)}^{3}-\mathcal{K}_{\sigma(32)}^{3}}{s_{2_{\sigma} 3_{\sigma}} s_{45}}+\frac{\mathcal{K}_{\sigma(23)}^{1}}{s_{3_{\sigma} 4} s_{51}} \\
& \mathcal{A}_{5}\left(1,2_{\sigma}, 4,3_{\sigma}, 5\right)=\frac{\mathcal{K}_{\sigma(23)}^{3}+\mathcal{K}_{\sigma(23)}^{2}}{s_{12_{\sigma}} s_{3_{\sigma} 5}}-\frac{\mathcal{K}_{\sigma(32)}^{1}}{s_{2_{\sigma} 4} s_{51}}+\frac{\mathcal{K}_{\sigma(23)}^{2}}{s_{3_{\sigma} s_{12}}}-\frac{\mathcal{K}_{\sigma(32)}^{2}+\mathcal{K}_{\sigma(32)}^{1}}{s_{3_{\sigma}{ } s_{2} s_{2}}}-\frac{\mathcal{K}_{\sigma(23)}^{1}}{s_{51} s_{3_{\sigma} 4}} \\
& \mathcal{A}_{5}\left(1,4,2_{\sigma}, 3_{\sigma}, 5\right)=\frac{\mathcal{K}_{\sigma(23)}^{3}+\mathcal{K}_{\sigma(23)}^{2}+\mathcal{K}_{\sigma(32)}^{2}+\mathcal{K}_{\sigma(32)}^{1}}{s_{14} s_{3 \sigma}}+\frac{\mathcal{K}_{\sigma(32)}^{1}}{s_{2_{\sigma}{ } s_{51}}} \\
& +\frac{-\mathcal{K}_{\sigma(32)}^{3}+\mathcal{K}_{\sigma(23)}^{3}-\mathcal{K}_{\sigma(23)}^{1}+\mathcal{K}_{\sigma(32)}^{1}}{s_{2_{\sigma} 3_{\sigma}} s_{14}}+\frac{-\mathcal{K}_{\sigma(32)}^{2}+\mathcal{K}_{\sigma(32)}^{1}}{s_{3_{\sigma} 5} s_{2_{\sigma} 4}}+\frac{-\mathcal{K}_{\sigma(23)}^{1}+\mathcal{K}_{\sigma(32)}^{1}}{s_{51} s_{2_{\sigma} 3_{\sigma}}}
\end{aligned}
$$

The last pair of color orderings $\mathcal{A}_{5}\left(1,4,2_{\sigma}, 3_{\sigma}, 5\right)$ has more complicated numerators because of the coefficient $P_{\left(4,2_{\sigma}, 3_{\sigma}\right)}^{\left(1,3_{\sigma}, 2_{\sigma}\right)}=\frac{1}{s_{14} s_{3} 5}+\operatorname{cyclic}\left(1,4,2_{\sigma}, 3_{\sigma}, 5\right)$ that addresses five different pole channels.

By comparing (4.4) with the $\mathcal{A}_{5}$ representation in the notation of [30],

$$
\mathcal{A}_{5}\left(1,2_{\rho}, 3_{\rho}, 4_{\rho}, 5\right)=\frac{n\left[12_{\rho}, 3_{\rho}, 4_{\rho} 5\right]}{s_{12_{\rho}} s_{4_{\rho} 5}}+\operatorname{cyclic}\left(1,2_{\rho}, 3_{\rho}, 4_{\rho}, 5\right)
$$


we can quickly read off the kinematic numerators:

$$
\begin{array}{rlrl}
n\left[12_{\sigma}, 3_{\sigma}, 45\right] & =\mathcal{K}_{\sigma(23)}^{3} & n\left[3_{\sigma} 4,5,2_{\sigma} 1\right] & =\mathcal{K}_{\sigma(23)}^{2} \\
n\left[2_{\sigma} 3_{\sigma}, 4,51\right] & =\mathcal{K}_{\sigma(23)}^{1}-\mathcal{K}_{\sigma(32)}^{1} & n\left[2_{\sigma} 3_{\sigma}, 1,45\right] & =\mathcal{K}_{\sigma(32)}^{3}-\mathcal{K}_{\sigma(23)}^{3} \\
n\left[3_{\sigma} 4,1,2_{\sigma} 5\right] & =\mathcal{K}_{\sigma(23)}^{1}+\mathcal{K}_{\sigma(23)}^{2} & n\left[12_{\sigma}, 4,3_{\sigma} 5\right] & =\mathcal{K}_{\sigma(23)}^{3}+\mathcal{K}_{\sigma(23)}^{2} \\
n\left[14,3_{\sigma}, 2_{\sigma} 5\right] & =\mathcal{K}_{\sigma(32)}^{3}+\mathcal{K}_{\sigma(23)}^{2}+\mathcal{K}_{\sigma(32)}^{2}+\mathcal{K}_{\sigma(23)}^{1} & & n\left[51,2_{\sigma}, 3_{\sigma} 4\right]=\mathcal{K}_{\sigma(23)}^{1} \\
n\left[2_{\sigma} 3_{\sigma}, 5,14\right] & =\mathcal{K}_{\sigma(23)}^{3}-\mathcal{K}_{\sigma(32)}^{3}+\mathcal{K}_{\sigma(32)}^{1}-\mathcal{K}_{\sigma(23)}^{1} &
\end{array}
$$

It is sufficient to display nine of them, the rest follows from $S_{2}$ relabelling $2 \leftrightarrow 3$. The $n_{1}, n_{2}, \ldots, n_{15}$ from the parametrization (3.3) translate into

$$
\begin{array}{lll}
n_{1}=n[12,3,45] & n_{6}=n[14,3,25] & n_{11}=n[24,3,51] \\
n_{2}=n[23,4,51] & n_{7}=n[32,5,14] & n_{12}=n[12,4,35] \\
n_{3}=n[34,5,12] & n_{8}=n[25,1,43] & n_{13}=n[35,1,24] \\
n_{4}=n[45,1,23] & n_{9}=n[13,4,25] & n_{14}=n[14,2,35] \\
n_{5}=n[51,2,34] & n_{10}=n[42,5,13] & n_{15}=n[13,2,45]
\end{array}
$$

The way in which the $n[i j, k, l m]$ are built out of $\mathcal{K}_{\sigma(2,3)}^{j}$ trivializes the Jacobi identities (3.4) or $n[i j,\{k, l m\}]=0$. However, the expressions (4.5) for $n[i j, k, l m]$ do not exhibit crossing symmetry including labels 1, 4 and 5 .

In many instances, the symmetry properties (2.11) of the BRST building blocks within $\mathcal{K}_{\sigma}^{l}$ allow to rewrite sums over several basic kinematics occurring in some $n_{i}$ as a single superfield, e.g.

$$
n_{2}=\mathcal{K}_{(23)}^{1}-\mathcal{K}_{(32)}^{1}=\left\langle\left(T_{123}-T_{132}\right) V_{4} V_{5}\right\rangle=\left\langle T_{321} V_{4} V_{5}\right\rangle
$$

However, the right hand side is outside the five point basis of kinematics, so the Jacobi relations between numerators are rather obscured by this building block manipulations. At any number of legs, the basis of $\mathcal{K}_{\sigma}^{l}$ is designed such that all the symmetries of the building blocks are already exploited, so we refrain from performing manipulations like $T_{123}-T_{132}=T_{321}$ in higher order examples.

\subsection{Six-point}

In six-point amplitudes, the propagator matrix (3.10) can be completely constructed from the field theory limit of the four superstring subamplitudes associated with color orderings $\{1,2,3,4,5,6\},\{1,2,3,5,4,6\},\{1,2,5,3,4,6\},\{1,5,2,3,4,6\}$. The $S_{3}$ relabelling 
covariance in 2,3,4 connects them to the remaining 20 elements of the KK basis. Let us give some representative sample entries of $P_{\rho}^{(l, \sigma)}$ here,

$$
\begin{aligned}
P_{(2345)}^{1,(423)} & =\frac{1}{s_{61} s_{23} s_{234}}, \quad P_{(2345)}{ }^{4,(432)}=\frac{1}{s_{56} s_{234}}\left(\frac{1}{s_{23}}+\frac{1}{s_{34}}\right) \\
P_{(2345)}{ }^{4,(234)} & =\frac{1}{s_{56}}\left(\frac{1}{s_{12} s_{34}}+\frac{1}{s_{12} s_{123}}+\frac{1}{s_{23} s_{123}}+\frac{1}{s_{23} s_{234}}+\frac{1}{s_{34} s_{234}}\right) \\
P_{(2354)}^{3,(234)} & =\frac{1}{s_{123}}\left(\frac{1}{s_{12}}+\frac{1}{s_{23}}\right)\left(\frac{1}{s_{45}}+\frac{1}{s_{46}}\right) \\
P_{(5234)}{ }^{1,(432)} & =\frac{1}{s_{61} s_{25} s_{34}}+\frac{1}{s_{15} s_{23} s_{46}}+\frac{1}{s_{15} s_{125} s_{34}}+\frac{1}{s_{125} s_{25} s_{34}}+\frac{1}{s_{15} s_{125} s_{46}} \\
& +\frac{1}{s_{46} s_{25} s_{125}}+\frac{1}{s_{15} s_{23} s_{234}}+\frac{1}{s_{61} s_{23} s_{234}}+\frac{1}{s_{15} s_{234} s_{34}}+\frac{1}{s_{61} s_{234} s_{34}} \\
& +\frac{1}{s_{61} s_{23} s_{235}}+\frac{1}{s_{61} s_{25} s_{235}}+\frac{1}{s_{46} s_{23} s_{235}}+\frac{1}{s_{46} s_{25} s_{235}}
\end{aligned}
$$

and refer the reader to Appendix A for the complete result.

Comparing the KK subamplitudes with

$$
\begin{gathered}
\mathcal{A}_{6}\left(1,2_{\rho}, 3_{\rho}, 4_{\rho}, 5_{\rho}, 6\right)=\frac{n\left[12_{\rho}, 3_{\rho} 4_{\rho}, 5_{\rho} 6\right]}{s_{12_{\rho}} s_{3_{\rho} 4_{\rho}} s_{5_{\rho} 6}}+\frac{n\left[2_{\rho} 3_{\rho}, 4_{\rho} 5_{\rho}, 61\right]}{s_{2_{\rho} 3_{\rho}} s_{4_{\rho} 5_{\rho}} s_{61}} \\
+\left(\frac{n\left[12_{\rho}, 3_{\rho}, 4_{\rho}, 5 \rho 6\right]}{s_{12_{\rho}} s_{12_{\rho} 3_{\rho}} s_{5} 6}-\frac{n\left[12_{\rho}, 3_{\rho}, 6,4_{\rho} 5_{\rho}\right]}{2 s_{12_{\rho}} s_{12_{\rho} 3_{\rho}} s_{4_{\rho} 5_{\rho}}}-\frac{n\left[2_{\rho} 3_{\rho}, 1,4_{\rho}, 5_{\rho} 6\right]}{2 s_{2_{\rho} 3_{\rho}} s_{12_{\rho} 3_{\rho}} s_{5 \rho} 6}+\operatorname{cyclic}\left(1,2_{\rho}, 3_{\rho}, 4_{\rho}, 5_{\rho}, 6\right)\right)
\end{gathered}
$$

allows to read off the 105 BCJ numerators. It is sufficient to display 25 of them in $S_{3^{-}}$ covariant form:

$$
\begin{array}{rlrl}
n\left[12_{\sigma}, 3_{\sigma}, 4_{\sigma}, 56\right] & =\mathcal{K}_{\sigma(234)}^{4} & n\left[61,2_{\sigma}, 3_{\sigma}, 54_{\sigma}\right] & =\mathcal{K}_{\sigma(234)}^{1} \\
n\left[12_{\sigma}, 3_{\sigma}, 6,4_{\sigma} 5\right] & =\mathcal{K}_{\sigma(234)}^{3} & n\left[12_{\sigma}, 6,3_{\sigma}, 54_{\sigma}\right] & =\mathcal{K}_{\sigma(234)}^{2} \\
n\left[12_{\sigma}, 3_{\sigma} 4_{\sigma}, 56\right] & =\mathcal{K}_{\sigma(234)}^{4}-\mathcal{K}_{\sigma(243)}^{4} & n\left[2_{\sigma} 3_{\sigma}, 4_{\sigma} 5,61\right] & =\mathcal{K}_{\sigma(324)}^{1}-\mathcal{K}_{\sigma(234)}^{1} \\
n\left[12_{\sigma}, 3_{\sigma} 5,64_{\sigma}\right] & =\mathcal{K}_{\sigma(243)}^{3}+\mathcal{K}_{\sigma(243)}^{2} & n\left[3_{\sigma} 4_{\sigma}, 5,6,12_{\sigma}\right] & =\mathcal{K}_{\sigma(234)}^{2}-\mathcal{K}_{\sigma(243)}^{2} \\
n\left[4_{\sigma} 5,6,1,2_{\sigma} 3_{\sigma}\right] & =\mathcal{K}_{\sigma(324)}^{3}-\mathcal{K}_{\sigma(234)}^{3} & n\left[3_{\sigma} 4_{\sigma}, 5,2_{\sigma}, 61\right] & =\mathcal{K}_{\sigma(234)}^{1}-\mathcal{K}_{\sigma(243)}^{1} \\
n\left[2_{\sigma} 3_{\sigma}, 1,4_{\sigma}, 56\right] & =\mathcal{K}_{\sigma(324)}^{4}-\mathcal{K}_{\sigma(234)}^{4} & n\left[12_{\sigma}, 3,5,5,46\right] & =\mathcal{K}_{\sigma(234)}^{4}+\mathcal{K}_{\sigma(234)}^{3} \\
n\left[4_{\sigma} 6,1,2_{\sigma}, 3_{\sigma} 5\right] & =\mathcal{K}_{\sigma(423)}^{2}+\mathcal{K}_{\sigma(423)}^{1} &
\end{array}
$$




$$
\begin{gathered}
n\left[2_{\sigma} 3_{\sigma}, 4_{\sigma}, 5,61\right]=-\mathcal{K}_{\sigma(234)}^{1}+\mathcal{K}_{\sigma(324)}^{1}+\mathcal{K}_{\sigma(423)}^{1}-\mathcal{K}_{\sigma(432)}^{1} \\
n\left[56,1,2_{\sigma}, 3_{\sigma} 4_{\sigma}\right]=\mathcal{K}_{\sigma(234)}^{4}-\mathcal{K}_{\sigma(243)}^{4}-\mathcal{K}_{\sigma(342)}^{4}+\mathcal{K}_{\sigma(432)}^{4} \\
n\left[2_{\sigma} 3_{\sigma}, 5,1,4_{\sigma} 6\right]=-\mathcal{K}_{\sigma(423)}^{2}+\mathcal{K}_{\sigma(432)}^{2}-\mathcal{K}_{\sigma(423)}^{1}+\mathcal{K}_{\sigma(432)}^{1} \\
n\left[2_{\sigma} 3_{\sigma}, 1,5,4_{\sigma} 6\right]=\mathcal{K}_{\sigma(234)}^{4}-\mathcal{K}_{\sigma(324)}^{4}+\mathcal{K}_{\sigma(234)}^{3}-\mathcal{K}_{\sigma(324)}^{3} \\
n\left[12_{\sigma}, 5,3_{\sigma}, 4_{\sigma} 6\right]=\mathcal{K}_{\sigma(234)}^{4}+\mathcal{K}_{\sigma(234)}^{3}+\mathcal{K}_{\sigma(243)}^{3}+\mathcal{K}_{\sigma(243)}^{2} \\
n\left[3_{\sigma} 4_{\sigma}, 6,1,2_{\sigma} 5\right]=-\mathcal{K}_{\sigma(342)}^{3}+\mathcal{K}_{\sigma(432)}^{3}+\mathcal{K}_{\sigma(342)}^{1}-\mathcal{K}_{\sigma(432)}^{1} \\
n\left[12_{\sigma}, 5,6,3_{\sigma} 4_{\sigma}\right]=-\mathcal{K}_{\sigma(234)}^{4}+\mathcal{K}_{\sigma(243)}^{4}+\mathcal{K}_{\sigma(234)}^{2}-\mathcal{K}_{\sigma(243)}^{2} \\
n\left[2_{\sigma} 5,1,3_{\sigma}, 4_{\sigma} 6\right]=\mathcal{K}_{\sigma(342)}^{3}+\mathcal{K}_{\sigma(342)}^{2}+\mathcal{K}_{\sigma(432)}^{2}+\mathcal{K}_{\sigma(432)}^{1} \\
n\left[15,2_{\sigma}, 3_{\sigma}, 4_{\sigma} 6\right]=\mathcal{K}_{\sigma(234)}^{4}+\mathcal{K}_{\sigma(234)}^{3}+\mathcal{K}_{\sigma(243)}^{3}+\mathcal{K}_{\sigma(342)}^{3}+\mathcal{K}_{\sigma(243)}^{2}+\mathcal{K}_{\sigma(342)}^{2}+\mathcal{K}_{\sigma(432)}^{2}+\mathcal{K}_{\sigma(432)}^{1} \\
n\left[23_{\sigma}, 4_{\sigma}, 6,15\right]=\mathcal{K}_{\sigma(234)}^{4}-\mathcal{K}_{\sigma(324)}^{4}-\mathcal{K}_{\sigma(423)}^{4}+\mathcal{K}_{\sigma(432)}^{4}+\mathcal{K}_{\sigma(234)}^{1}-\mathcal{K}_{\sigma(324)}^{1}-\mathcal{K}_{\sigma(423)}^{1}+\mathcal{K}_{\sigma(432)}^{1} \\
n\left[15,2_{\sigma}, 6,3_{\sigma} 4_{\sigma}\right]=\mathcal{K}_{\sigma(243)}^{4}-\mathcal{K}_{\sigma(234)}^{4}-\mathcal{K}_{\sigma(342)}^{3}+\mathcal{K}_{\sigma(432)}^{3}+\mathcal{K}_{\sigma(234)}^{2}-\mathcal{K}_{\sigma(243)}^{2}+\mathcal{K}_{\sigma(342)}^{1}-\mathcal{K}_{\sigma(432)}^{1} \\
n\left[15,2_{\sigma} 3_{\sigma}, 4_{\sigma} 6\right]=\mathcal{K}_{\sigma(234)}^{4}-\mathcal{K}_{\sigma(324)}^{4}+\mathcal{K}_{\sigma(234)}^{3}-\mathcal{K}_{\sigma(324)}^{3}-\mathcal{K}_{\sigma(423)}^{2}+\mathcal{K}_{\sigma(432)}^{2}-\mathcal{K}_{\sigma(423)}^{1}+\mathcal{K}_{\sigma(432)}^{1}
\end{gathered}
$$

They have been explicitly checked to satisfy all the 105 Jacobi relations $n[i j, k,\{l, m p\}]=0$ and $n[i j, k l, m p]=n[i j, k, l, m p]-n[i j, l, k, m p]$ (81 of which are linearly independent). It is interesting to note that the number of $K_{\sigma}^{l}$ forming the individual BCJ numerators is always a power of two, i.e. 1,2, 4 or 8 in this case.

\subsection{Seven-point}

Since the number of channels grows like $(2 n-5)$ !! in an $n$-point amplitude, a complete list of all BCJ numerators becomes very lengthy beyond six points. Appendix B gives all the 69 seven-point numerators which are not related by 2,3,4,5 relabelling, they allow to

obtain all the 945 numerators by going through the $\sigma \in S_{4}$ permutations of $(2,3,4,5)$. We also checked that all the 825 independent numerators equations (out of 1260 in total) are satisfied.

\subsection{Higher-point and general observations}

Instead of continuing the numerator list to higher points, we conclude this section with some general remarks and observation on the structure of the string inspired expressions for the BCJ numerators. 
Firstly, entries of the $n$ point propagator matrix always factorize into sums of $m$ propagators with $C(m)=\frac{(2 m) !}{m !(m+1) !}$ terms,

$$
P_{\rho}^{(l, \sigma)} \sim \prod\left(\sum_{j=1}^{C(m)} \frac{1}{s_{\left(\alpha^{1}\right)_{j}} s_{\left(\alpha^{2}\right)_{j}} \ldots s_{\left(\alpha^{m}\right)_{j}}}\right)
$$

where $C(m)$ is the $m$ 'th Catalan number and counts the number of channels appearing in a $m+2$ point color ordered amplitude [31]. At $n=5$, we have seen three different pole structures in (4.4),

$$
\left.P_{\rho}^{(l, \sigma)}\right|_{n=5} \sim\left\{\frac{1}{s_{\alpha} s_{\beta}}, \frac{1}{s_{\alpha}}\left(\frac{1}{s_{\beta_{1}}}+\frac{1}{s_{\beta_{2}}}\right), \sum_{i=1}^{5} \frac{1}{s_{\alpha_{i}} s_{\beta_{i}}}\right\}
$$

and the six point analogue contains the five types of products displayed in (4.6):

$$
\begin{aligned}
& \left.P_{\rho}^{(l, \sigma)}\right|_{n=6} \sim\left\{\frac{1}{s_{\alpha} s_{\beta} s_{\gamma}}, \frac{1}{s_{\alpha} s_{\beta}}\left(\frac{1}{s_{\gamma_{1}}}+\frac{1}{s_{\gamma_{2}}}\right), \frac{1}{s_{\alpha}}\left(\frac{1}{s_{\beta_{1}}}+\frac{1}{s_{\beta_{2}}}\right)\left(\frac{1}{s_{\gamma_{1}}}+\frac{1}{s_{\gamma_{2}}}\right),\right. \\
& \left.\frac{1}{s_{\alpha}} \sum_{i=1}^{5} \frac{1}{s_{\beta_{i}} s_{\gamma_{i}}}, \sum_{j=1}^{14} \frac{1}{s_{\alpha_{j}} s_{\beta_{j}} s_{\gamma_{j}}}\right\}
\end{aligned}
$$

The pattern was observed to persist up to eight-point. However, not all possible partitions of the overall $n-3$ propagators into products of type (4.8) are realized. For instance, there are no terms like $\left(\frac{1}{s_{\alpha_{1}}}+\frac{1}{s_{\alpha_{2}}}\right)\left(\frac{1}{s_{\beta_{1}}}+\frac{1}{s_{\beta_{2}}}\right)$ at five points, $\left(\frac{1}{s_{\alpha_{1}}}+\frac{1}{s_{\alpha_{2}}}\right)\left(\sum_{i=1}^{5} \frac{1}{s_{\beta_{i}} s_{\gamma_{i}}}\right)$ at six points and $\left(\frac{1}{s_{\alpha_{1}}}+\frac{1}{s_{\alpha_{2}}}\right)\left(\frac{1}{s_{\beta_{1}}}+\frac{1}{s_{\beta_{2}}}\right)\left(\frac{1}{s_{\gamma_{1}}}+\frac{1}{s_{\gamma_{2}}}\right) \frac{1}{s_{\delta}}$ at seven points.

Secondly, the number of $\mathcal{K}_{\sigma}^{l}$ kinematics entering the individual $n$ point BCJ numerators up to $n=8$ is always a power of two $1,2,4, \ldots, 2^{n-3}$. This can be largely explained from the flipping antisymmetry of $n[i j, k, \ldots]$ in pairs of labels $i, j$ sharing a terminal three point vertex. If they are both from the range $i_{\sigma}, j_{\sigma} \in\{2,3, \ldots, n-2\}$, then the $\mathcal{K}_{\sigma}^{l}$ are required to pair up with their $i \leftrightarrow j$ images. Moreover, if several other $2,3, \ldots, n-2$ labels $k_{\sigma}, l_{\sigma}$ follow, then a nested antisymmetrization emerges, e.g. $n\left[i_{\sigma} j_{\sigma}, k_{\sigma}, l_{\sigma}, \ldots\right] \leftrightarrow \mathcal{K}_{\sigma\left(\left[\left[\left[i_{\sigma} j_{\sigma}\right] k_{\sigma}\right] l_{\sigma}\right] \ldots\right)}^{l}$

Another source of doubling the terms is a terminal vertex with legs 1 and $n-1$ : Swapping $1 \leftrightarrow n-1$ maps $\mathcal{K}_{\sigma}^{l}$ to $\mathcal{K}_{\bar{\sigma}}^{n-1-l}$ where $\bar{\sigma}$ denotes the permutation of reverse order, $\bar{\sigma}(23 \ldots p-1, p)=\sigma(p, p-1, \ldots 32)$. That is why $n[1(n-1), \ldots]$ can only contain pairs like $\mathcal{K}_{\sigma}^{l}+\mathcal{K}_{\bar{\sigma}}^{n-1-l}$ which might be further antisymmetrized in some legs from $\{2,3, \ldots, n-2\}$ due to another terminal vertex. 
The following table shows the distribution of the $(2 n-5) ! !$ numerators into packages of $2^{j}$ basis elements:

\begin{tabular}{|c||c|c|c|c|c|}
\hline \# terms & 4 pts & $5 p t s$ & 6 pts & 7 pts & 8 pts \\
\hline \hline 1 & 2 & 6 & 24 & 120 & 720 \\
\hline 2 & 1 & 6 & 36 & 240 & 1800 \\
\hline 4 & & 3 & 30 & 270 & 2520 \\
\hline 8 & & & 15 & 210 & 2520 \\
\hline 16 & & & & 105 & 1890 \\
\hline 32 & & & & & 945 \\
\hline
\end{tabular}

Table 1. The number of BCJ numerators in $n$-point amplitudes containing $2^{j}$ basis kinematics $\mathcal{K}_{\sigma}^{l}$ for $j=0,1, \ldots, n-3$.

We suspect that the grading of kinematic numerators according to their $\mathcal{K}_{\sigma}^{l}$ content is connected with the factorization pattern $(4.8)$ of $P_{\rho}^{(l, \sigma)}$ entries.

\section{Concluding remarks}

In this paper we have developed a method based on string theory to construct kinematic factors $n_{i}$ for gauge theory amplitudes which manifestly obey Jacobi identities dual to the color algebra $c_{i}+c_{j}+c_{k}=0$. The fact that the vanishing of the dual numerator triplet $n_{i}+n_{j}+n_{k}$ depends on the organization of contact terms complicates the direct construction of $n_{i}$ within the gauge theory setup.

The pure spinor approach to superstring theory naturally introduces a kinematic basis (3.9) of $(n-2)$ ! elements for $n$-point tree amplitudes of the gauge multiplet. The field theory amplitude can be extracted by taking the low energy limit of the string result (3.5) using the method of [5]. This determines the BCJ numerators $n_{i}$ for any pole channel in term of the $(n-2)$ ! basis kinematics. The resulting expressions for the $n_{i}$ are manifestly local and supersymmetric. Although they originate from the ten dimensional SYM theory, it is still straightforward to dimensionally reduce the superfield components and to recycle the purely bosonic amplitudes for QCD or any other theory with less than sixteen supercharges.

The basis dimension $(n-2)$ ! together with the BCJ relations between color ordered field theory amplitudes imply that the string inspired $n_{i}$ satisfy the dual Jacobi identities $n_{i}+n_{j}+n_{k}=0$ for each vanishing triplet of color factors $\left(c_{i}, c_{j}, c_{k}\right)$. However, as a price to pay for the exact $n_{i} \leftrightarrow c_{i}$ duality, crossing symmetry is lost for the kinematic numerators. 
This can be immediately recognized from the explicit solutions (4.5), (4.7) and appendix $\mathrm{B}$ for the $n_{i}$ at five, six and seven points, respectively. It would be interesting to find a compact form for crossing symmetric numerators while still preserving the Jacobi-like relations.

\section{Acknowledgments}

We thank the Kavli Institute for Theoretical Physics in Santa Barbara for hospitality and financial support during preparation and completion of this work. We are indebted to Zvi Bern for initiating this work and for helpful discussions. Furthermore, we are grateful to John Joseph Carrasco and Henrik Johansson for strong encouragement, stimulating discussions and for contributing valuable suggestions to the manuscript. This research was supported in part by the National Science Foundation under Grant No. NSF PHY0551164. C.M. thanks the partial financial support from the MPG and acknowledges support by the Deutsch-Israelische Projektkooperation (DIP H52).

\section{Appendix A. Field theory limit of six-point integrals}

This appendix contains the field theory limit of the six-point superstring amplitudes in the KK color orderings. It is the higher point analogue of the five point result (4.4).

$$
\begin{aligned}
& \mathcal{A}\left(1,2_{\sigma}, 3_{\sigma}, 4_{\sigma}, 5,6\right)=\frac{\mathcal{K}_{\sigma(234)}^{3}}{s_{12_{\sigma}} s_{4_{\sigma} 5} s_{12_{\sigma} 3_{\sigma}}}+\frac{-\mathcal{K}_{\sigma(234)}^{2}}{s_{12_{\sigma}} s_{4_{\sigma} 5} s_{3_{\sigma} 4_{\sigma} 5}} \\
& +\frac{-\mathcal{K}_{\sigma(234)}^{4}}{s_{12_{\sigma}} s_{56} s_{12_{\sigma} 3_{\sigma}}}+\frac{\mathcal{K}_{\sigma(234)}^{1}}{s_{16} s_{4_{\sigma} 5} s_{3_{\sigma} 4_{\sigma} 5}}+\frac{-\mathcal{K}_{\sigma(234)}^{4}+\mathcal{K}_{\sigma(243)}^{4}}{s_{12_{\sigma}} s_{3_{\sigma} 4_{\sigma}} s_{56}}+\frac{-\mathcal{K}_{\sigma(234)}^{2}+\mathcal{K}_{\sigma(243)}^{2}}{s_{12_{\sigma}} s_{3_{\sigma} 4_{\sigma}} s_{3_{\sigma} 4_{\sigma} 5}} \\
& +\frac{\mathcal{K}_{\sigma(234)}^{1}-\mathcal{K}_{\sigma(324)}^{1}}{s_{16} s_{2_{\sigma} 3_{\sigma}} s_{4_{\sigma} 5}}+\frac{\mathcal{K}_{\sigma(234)}^{1}-\mathcal{K}_{\sigma(243)}^{1}}{s_{16} s_{3_{\sigma} 4_{\sigma}} s_{3_{\sigma} 4_{\sigma} 5}}+\frac{\mathcal{K}_{\sigma(234)}^{3}-\mathcal{K}_{\sigma(324)}^{3}}{s_{2_{\sigma} 3_{\sigma}} s_{4_{\sigma} 5} s_{12_{\sigma} 3_{\sigma}}}+\frac{-\mathcal{K}_{\sigma(234)}^{4}+\mathcal{K}_{\sigma(324)}^{4}}{s_{2_{\sigma} 3_{\sigma}} s_{56} s_{12_{\sigma} 3_{\sigma}}} \\
& +\frac{\mathcal{K}_{\sigma(234)}^{1}-\mathcal{K}_{\sigma(324)}^{1}-\mathcal{K}_{\sigma(423)}^{1}+\mathcal{K}_{\sigma(432)}^{1}}{s_{16} s_{2_{\sigma} 3_{\sigma}} s_{2_{\sigma} 3_{\sigma} 4_{\sigma}}}+\frac{\mathcal{K}_{\sigma(234)}^{1}-\mathcal{K}_{\sigma(243)}^{1}-\mathcal{K}_{\sigma(342)}^{1}+\mathcal{K}_{\sigma(432)}^{1}}{s_{16} s_{3_{\sigma} 4_{\sigma}} s_{2_{\sigma} 3_{\sigma} 4_{\sigma}}}
\end{aligned}
$$

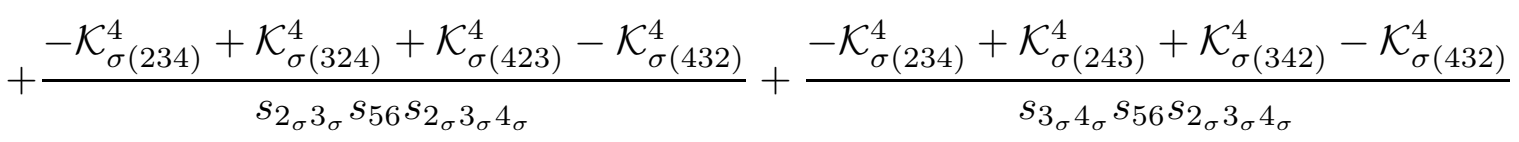

$$
\begin{aligned}
& \mathcal{A}\left(1,2_{\sigma}, 3_{\sigma}, 5,4_{\sigma}, 6\right)=\frac{\mathcal{K}_{\sigma(243)}^{2}}{s_{12_{\sigma}} s_{3_{\sigma} 5} s_{3_{\sigma} 4_{\sigma} 5}}+\frac{-\mathcal{K}_{\sigma(234)}^{3}}{s_{12_{\sigma} s_{4_{\sigma} 5} s_{12_{\sigma} 3_{\sigma}}}}+\frac{\mathcal{K}_{\sigma(234)}^{2}}{s_{12_{\sigma} s_{4_{\sigma} 5} s_{3_{\sigma} 4_{\sigma} 5}}}
\end{aligned}
$$




$$
\begin{aligned}
& +\frac{-\mathcal{K}_{\sigma(423)}^{1}}{s_{16} s_{3_{\sigma} 5} s_{2_{\sigma} 3_{\sigma} 5}}+\frac{-\mathcal{K}_{\sigma(243)}^{1}}{s_{16} s_{3_{\sigma} 5} s_{3_{\sigma} 4_{\sigma} 5}}+\frac{-\mathcal{K}_{\sigma(234)}^{1}}{s_{16} s_{4_{\sigma} 5} s_{3_{\sigma} 4_{\sigma} 5}}+\frac{\mathcal{K}_{\sigma(243)}^{3}+\mathcal{K}_{\sigma(243)}^{2}}{s_{12_{\sigma}} s_{3_{\sigma} 5} s_{4_{\sigma} 6}}+\frac{-\mathcal{K}_{\sigma(234)}^{4}-\mathcal{K}_{\sigma(234)}^{3}}{s_{12_{\sigma}} s_{4_{\sigma} 6} s_{12_{\sigma} 3_{\sigma}}} \\
& +\frac{-\mathcal{K}_{\sigma(234)}^{1}+\mathcal{K}_{\sigma(324)}^{1}}{s_{16} s_{2_{\sigma} 3_{\sigma}} s_{4_{\sigma} 5}}+\frac{-\mathcal{K}_{\sigma(423)}^{1}+\mathcal{K}_{\sigma(432)}^{1}}{s_{16} s_{2_{\sigma} 3_{\sigma}} s_{2_{\sigma} 3_{\sigma} 5}}+\frac{-\mathcal{K}_{\sigma(234)}^{3}+\mathcal{K}_{\sigma(324)}^{3}}{s_{2_{\sigma} 3_{\sigma}} s_{4_{\sigma} 5} s_{12_{\sigma} 3_{\sigma}}}+\frac{-\mathcal{K}_{\sigma(423)}^{2}-\mathcal{K}_{\sigma(423)}^{1}}{s_{3_{\sigma} 5} s_{4_{\sigma} 6} s_{2_{\sigma} 3_{\sigma} 5}} \\
& +\frac{-\mathcal{K}_{\sigma(234)}^{4}+\mathcal{K}_{\sigma(324)}^{4}-\mathcal{K}_{\sigma(234)}^{3}+\mathcal{K}_{\sigma(324)}^{3}}{s_{2_{\sigma} 3_{\sigma} s_{4_{\sigma} 6} s_{12_{\sigma} 3_{\sigma}}}}+\frac{-\mathcal{K}_{\sigma(423)}^{2}+\mathcal{K}_{\sigma(432)}^{2}-\mathcal{K}_{\sigma(423)}^{1}+\mathcal{K}_{\sigma(432)}^{1}}{s_{2_{\sigma} 3_{\sigma}} s_{4_{\sigma} 6} s_{2_{\sigma} 3_{\sigma} 5}} \\
& \mathcal{A}\left(1,2_{\sigma}, 5,3_{\sigma}, 4_{\sigma}, 6\right)=\frac{-\mathcal{K}_{\sigma(243)}^{2}}{s_{12_{\sigma}} s_{3_{\sigma} 5} s_{3_{\sigma} 4_{\sigma} 5}}+\frac{\mathcal{K}_{\sigma(432)}^{1}}{s_{16} s_{2_{\sigma} 5} s_{2_{\sigma} 3_{\sigma} 5}} \\
& +\frac{\mathcal{K}_{\sigma(423)}^{1}}{s_{16} s_{3_{\sigma} 5} s_{2_{\sigma} 3_{\sigma} 5}}+\frac{\mathcal{K}_{\sigma(243)}^{1}}{s_{16} s_{3_{\sigma} 5} s_{3_{\sigma} 4_{\sigma} 5}}+\frac{-\mathcal{K}_{\sigma(342)}^{1}+\mathcal{K}_{\sigma(432)}^{1}}{s_{16} s_{2_{\sigma} 5} s_{3_{\sigma} 4_{\sigma}}}+\frac{-\mathcal{K}_{\sigma(234)}^{1}+\mathcal{K}_{\sigma(243)}^{1}}{s_{16} s_{3_{\sigma} 4_{\sigma}} s_{3_{\sigma} 4_{\sigma} 5}} \\
& +\frac{\mathcal{K}_{\sigma(234)}^{2}-\mathcal{K}_{\sigma(243)}^{2}}{s_{12_{\sigma} s_{3_{\sigma} 4_{\sigma}} s_{3_{\sigma} 4_{\sigma} 5}}}+\frac{-\mathcal{K}_{\sigma(243)}^{3}-\mathcal{K}_{\sigma(243)}^{2}}{s_{12_{\sigma} s_{3_{\sigma} 5} s_{4_{\sigma}}}}+\frac{\mathcal{K}_{\sigma(432)}^{2}+\mathcal{K}_{\sigma(432)}^{1}}{s_{2_{\sigma} 5} s_{4_{\sigma} 6} s_{2_{\sigma} 3_{\sigma} 5}}+\frac{\mathcal{K}_{\sigma(423)}^{2}+\mathcal{K}_{\sigma(423)}^{1}}{s_{3_{\sigma} 5} s_{4_{\sigma}{ }} s_{2_{\sigma} 3_{\sigma} 5}} \\
& +\frac{-\mathcal{K}_{\sigma(234)}^{4}-\mathcal{K}_{\sigma(234)}^{3}-\mathcal{K}_{\sigma(243)}^{3}-\mathcal{K}_{\sigma(243)}^{2}}{s_{12_{\sigma}} s_{4_{\sigma} 6} s_{12_{\sigma} 5}}+\frac{-\mathcal{K}_{\sigma(234)}^{4}+\mathcal{K}_{\sigma(243)}^{4}+\mathcal{K}_{\sigma(234)}^{2}-\mathcal{K}_{\sigma(243)}^{2}}{s_{12_{\sigma}} s_{3_{\sigma} 4_{\sigma}} s_{12_{\sigma} 5}} \\
& +\frac{\mathcal{K}_{\sigma(342)}^{3}-\mathcal{K}_{\sigma(432)}^{3}-\mathcal{K}_{\sigma(342)}^{1}+\mathcal{K}_{\sigma(432)}^{1}}{s_{2_{\sigma} 5} s_{3_{\sigma} 4_{\sigma}} s_{12_{\sigma} 5}}+\frac{\mathcal{K}_{\sigma(342)}^{3}+\mathcal{K}_{\sigma(342)}^{2}+\mathcal{K}_{\sigma(432)}^{2}+\mathcal{K}_{\sigma(432)}^{1}}{s_{2_{\sigma}{ } s_{4_{\sigma}} s_{12} s_{1} 5}} \\
& \mathcal{A}\left(1,5,2_{\sigma}, 3_{\sigma}, 4_{\sigma}, 6\right)=\frac{-\mathcal{K}_{\sigma(342)}^{3}-\mathcal{K}_{\sigma(342)}^{2}-\mathcal{K}_{\sigma(432)}^{2}-\mathcal{K}_{\sigma(432)}^{1}}{s_{2_{\sigma} 5} s_{4_{\sigma} 6} s_{12_{\sigma} 5}} \\
& +\frac{-\mathcal{K}_{\sigma(234)}^{4}+\mathcal{K}_{\sigma(324)}^{4}-\mathcal{K}_{\sigma(234)}^{3}+\mathcal{K}_{\sigma(324)}^{3}+\mathcal{K}_{\sigma(423)}^{2}-\mathcal{K}_{\sigma(432)}^{2}+\mathcal{K}_{\sigma(423)}^{1}-\mathcal{K}_{\sigma(432)}^{1}}{s_{15} s_{2_{\sigma} 3_{\sigma}} s_{4_{\sigma} 6}} \\
& +\frac{-\mathcal{K}_{\sigma(234)}^{4}+\mathcal{K}_{\sigma(324)}^{4}+\mathcal{K}_{\sigma(423)}^{4}-\mathcal{K}_{\sigma(432)}^{4}-\mathcal{K}_{\sigma(234)}^{1}+\mathcal{K}_{\sigma(324)}^{1}+\mathcal{K}_{\sigma(423)}^{1}-\mathcal{K}_{\sigma(432)}^{1}}{s_{15} s_{2_{\sigma} 3_{\sigma}} s_{2_{\sigma} 3_{\sigma} 4_{\sigma}}} \\
& +\frac{-\mathcal{K}_{\sigma(234)}^{4}+\mathcal{K}_{\sigma(243)}^{4}-\mathcal{K}_{\sigma(342)}^{3}+\mathcal{K}_{\sigma(432)}^{3}+\mathcal{K}_{\sigma(234)}^{2}-\mathcal{K}_{\sigma(243)}^{2}+\mathcal{K}_{\sigma(342)}^{1}-\mathcal{K}_{\sigma(432)}^{1}}{s_{15} s_{3_{\sigma} 4_{\sigma}} s_{12_{\sigma} 5}} \\
& +\frac{-\mathcal{K}_{\sigma(234)}^{4}+\mathcal{K}_{\sigma(243)}^{4}+\mathcal{K}_{\sigma(342)}^{4}-\mathcal{K}_{\sigma(432)}^{4}-\mathcal{K}_{\sigma(234)}^{1}+\mathcal{K}_{\sigma(243)}^{1}+\mathcal{K}_{\sigma(342)}^{1}-\mathcal{K}_{\sigma(432)}^{1}}{s_{15} s_{3_{\sigma} 4_{\sigma}} s_{2_{\sigma} 3_{\sigma} 4_{\sigma}}} \\
& +\frac{-\mathcal{K}_{\sigma(234)}^{4}-\mathcal{K}_{\sigma(234)}^{3}-\mathcal{K}_{\sigma(243)}^{3}-\mathcal{K}_{\sigma(342)}^{3}-\mathcal{K}_{\sigma(243)}^{2}-\mathcal{K}_{\sigma(342)}^{2}-\mathcal{K}_{\sigma(432)}^{2}-\mathcal{K}_{\sigma(432)}^{1}}{s_{15} s_{4 \sigma} s_{122_{\sigma}}} \\
& +\frac{-\mathcal{K}_{\sigma(432)}^{1}}{s_{16} s_{2_{\sigma} 5} s_{2_{\sigma} 3_{\sigma} 5}}+\frac{\mathcal{K}_{\sigma(423)}^{1}-\mathcal{K}_{\sigma(432)}^{1}}{s_{16} s_{2_{\sigma} 3_{\sigma}} s_{2_{\sigma} 3_{\sigma} 5}}+\frac{\mathcal{K}_{\sigma(342)}^{1}-\mathcal{K}_{\sigma(432)}^{1}}{s_{16} s_{2_{\sigma} 5} s_{3_{\sigma} 4_{\sigma}}}+\frac{-\mathcal{K}_{\sigma(432)}^{2}-\mathcal{K}_{\sigma(432)}^{1}}{s_{2_{\sigma} 5} s_{4_{\sigma} 6} s_{2_{\sigma} 3_{\sigma} 5}} \\
& +\frac{-\mathcal{K}_{\sigma(234)}^{1}+\mathcal{K}_{\sigma(324)}^{1}+\mathcal{K}_{\sigma(423)}^{1}-\mathcal{K}_{\sigma(432)}^{1}}{s_{16} s_{2_{\sigma} 3_{\sigma}} s_{2_{\sigma} 3_{\sigma} 4_{\sigma}}}+\frac{-\mathcal{K}_{\sigma(234)}^{1}+\mathcal{K}_{\sigma(243)}^{1}+\mathcal{K}_{\sigma(342)}^{1}-\mathcal{K}_{\sigma(432)}^{1}}{s_{16} s_{3_{\sigma} 4_{\sigma}} s_{2_{\sigma} 3_{\sigma} 4_{\sigma}}}
\end{aligned}
$$




$$
+\frac{\mathcal{K}_{\sigma(423)}^{2}-\mathcal{K}_{\sigma(432)}^{2}+\mathcal{K}_{\sigma(423)}^{1}-\mathcal{K}_{\sigma(432)}^{1}}{s_{2_{\sigma} 3_{\sigma}} s_{4_{\sigma} 6} s_{2_{\sigma} 3_{\sigma} 5}}+\frac{-\mathcal{K}_{\sigma(342)}^{3}+\mathcal{K}_{\sigma(432)}^{3}+\mathcal{K}_{\sigma(342)}^{1}-\mathcal{K}_{\sigma(432)}^{1}}{s_{2_{\sigma} 5} s_{3_{\sigma} 4_{\sigma}} s_{12_{\sigma} 5}}
$$

Note that the basis kinematics $\mathcal{K}_{\sigma(432)}^{1}$ contributes to each of the fourteen pole channels of $\mathcal{A}_{6}\left(1,5,2_{\sigma}, 3_{\sigma}, 4_{\sigma}, 6\right)$.

\section{Appendix B. Seven-point numerators}

This appendix lists the kinematic numerators for the seven-point amplitude. Thanks to $S_{4}$ covariance in $(2,3,4,5)$ permutations, only 69 out of the 945 BCJ numerators have to be displayed explicitly, the rest follows from relabelling of legs $2,3,4$ and 5 :

$$
\begin{aligned}
& n\left[12_{\sigma}, 3_{\sigma}, 4_{\sigma}, 5_{\sigma}, 67\right]=\mathcal{K}_{\sigma(2345)}^{5} \\
& n\left[71,2_{\sigma}, 3_{\sigma}, 4_{\sigma}, 5_{\sigma} 6\right]=\mathcal{K}_{\sigma(2345)}^{1} \\
& n\left[12_{\sigma}, 7,3_{\sigma}, 4_{\sigma}, 5_{\sigma} 6\right]=\mathcal{K}_{\sigma(2345)}^{2} \\
& n\left[12_{\sigma}, 3_{\sigma}, 4_{\sigma} 5_{\sigma}, 67\right]=\mathcal{K}_{\sigma(2345)}^{5}-\mathcal{K}_{\sigma(2354)}^{5} \\
& n\left[12_{\sigma}, 7,3_{\sigma}, 6,4_{\sigma} 5_{\sigma}\right]=\mathcal{K}_{\sigma(2354)}^{2}-\mathcal{K}_{\sigma(2345)}^{2} \\
& n\left[5_{\sigma} 6,7,12_{\sigma}, 3_{\sigma} 4_{\sigma}\right]=\mathcal{K}_{\sigma(2435)}^{4}-\mathcal{K}_{\sigma(2345)}^{4} \\
& n\left[12_{\sigma}, 3_{\sigma}, 4_{\sigma} 6,75_{\sigma}\right]=\mathcal{K}_{\sigma(2354)}^{3}+\mathcal{K}_{\sigma(2354)}^{4} \\
& n\left[12_{\sigma}, 7,3_{\sigma} 4_{\sigma}, 5_{\sigma} 6\right]=\mathcal{K}_{\sigma(2345)}^{2}-\mathcal{K}_{\sigma(2435)}^{2} \\
& n\left[4_{\sigma} 5_{\sigma}, 6,7,3_{\sigma}, 12_{\sigma}\right]=\mathcal{K}_{\sigma(2354)}^{3}-\mathcal{K}_{\sigma(2345)}^{3} \\
& n\left[5_{\sigma} 6,4_{\sigma}, 7,1,2_{\sigma} 3_{\sigma}\right]=\mathcal{K}_{\sigma(3245)}^{3}-\mathcal{K}_{\sigma(2345)}^{3} \\
& n\left[67,5_{\sigma}, 12_{\sigma}, 3_{\sigma} 4_{\sigma}\right]=\mathcal{K}_{\sigma(2435)}^{5}-\mathcal{K}_{\sigma(2345)}^{5} \\
& n\left[4_{\sigma} 5_{\sigma}, 6,71,2_{\sigma} 3_{\sigma}\right]=\mathcal{K}_{\sigma(2345)}^{1}-\mathcal{K}_{\sigma(2354)}^{1}-\mathcal{K}_{\sigma(3245)}^{1}+\mathcal{K}_{\sigma(3254)}^{1} \\
& n\left[4_{\sigma} 5_{\sigma}, 6,7,1,2_{\sigma} 3_{\sigma}\right]=\mathcal{K}_{\sigma(2345)}^{3}-\mathcal{K}_{\sigma(2354)}^{3}-\mathcal{K}_{\sigma(3245)}^{3}+\mathcal{K}_{\sigma(3254)}^{3} \\
& n\left[2_{\sigma} 3_{\sigma}, 1,4_{\sigma} 5_{\sigma}, 67\right]=\mathcal{K}_{\sigma(2354)}^{5}-\mathcal{K}_{\sigma(2345)}^{5}+\mathcal{K}_{\sigma(3245)}^{5}-\mathcal{K}_{\sigma(3254)}^{5} \\
& n\left[2_{\sigma} 3_{\sigma}, 4_{\sigma}, 5_{\sigma} 6,71\right]=\mathcal{K}_{\sigma(2345)}^{1}-\mathcal{K}_{\sigma(3245)}^{1}-\mathcal{K}_{\sigma(4235)}^{1}+\mathcal{K}_{\sigma(4325)}^{1} \\
& n\left[3_{\sigma} 4_{\sigma}, 5_{\sigma}, 6,7,12_{\sigma}\right]=\mathcal{K}_{\sigma(2435)}^{2}-\mathcal{K}_{\sigma(2345)}^{2}+\mathcal{K}_{\sigma(2534)}^{2}-\mathcal{K}_{\sigma(2543)}^{2} \\
& n\left[3_{\sigma} 4_{\sigma}, 5_{\sigma}, 67,12_{\sigma}\right]=\mathcal{K}_{\sigma(2345)}^{5}-\mathcal{K}_{\sigma(2435)}^{5}-\mathcal{K}_{\sigma(2534)}^{5}+\mathcal{K}_{\sigma(2543)}^{5} \\
& n\left[3_{\sigma} 4_{\sigma}, 6,5_{\sigma} 7,12_{\sigma}\right]=\mathcal{K}_{\sigma(2534)}^{2}-\mathcal{K}_{\sigma(2543)}^{2}+\mathcal{K}_{\sigma(2534)}^{3}-\mathcal{K}_{\sigma(2543)}^{3} \\
& n\left[4_{\sigma} 5_{\sigma}, 7,1,2_{\sigma}, 3_{\sigma} 6\right]=\mathcal{K}_{\sigma(5423)}^{1}-\mathcal{K}_{\sigma(4523)}^{1}+\mathcal{K}_{\sigma(4523)}^{3}-\mathcal{K}_{\sigma(5423)}^{3} \\
& n\left[4_{\sigma} 5_{\sigma}, 7,12_{\sigma}, 3_{\sigma} 6\right]=\mathcal{K}_{\sigma(2453)}^{2}-\mathcal{K}_{\sigma(2543)}^{2}-\mathcal{K}_{\sigma(2453)}^{4}+\mathcal{K}_{\sigma(2543)}^{4} \\
& n\left[5_{\sigma} 6,7,1,2_{\sigma}, 3_{\sigma} 4_{\sigma}\right]=\mathcal{K}_{\sigma(2435)}^{4}-\mathcal{K}_{\sigma(2345)}^{4}+\mathcal{K}_{\sigma(3425)}^{4}-\mathcal{K}_{\sigma(4325)}^{4} \\
& n\left[5_{\sigma} 7,1,2_{\sigma} 3_{\sigma}, 4_{\sigma} 6\right]=\mathcal{K}_{\sigma(5324)}^{1}-\mathcal{K}_{\sigma(5234)}^{1}-\mathcal{K}_{\sigma(5234)}^{2}+\mathcal{K}_{\sigma(5324)}^{2} \\
& n\left[12_{\sigma}, 3_{\sigma}, 6,4_{\sigma}, 5_{\sigma} 7\right]=\mathcal{K}_{\sigma(2354)}^{3}+\mathcal{K}_{\sigma(2345)}^{4}+\mathcal{K}_{\sigma(2354)}^{4}+\mathcal{K}_{\sigma(2345)}^{5} \\
& n\left[12_{\sigma}, 3_{\sigma}, 6,7,4_{\sigma} 5_{\sigma}\right]=\mathcal{K}_{\sigma(2345)}^{3}-\mathcal{K}_{\sigma(2354)}^{3}-\mathcal{K}_{\sigma(2345)}^{5}+\mathcal{K}_{\sigma(2354)}^{5}
\end{aligned}
$$




$$
\begin{aligned}
& n\left[2_{\sigma} 3_{\sigma}, 1,4_{\sigma} 6,5_{\sigma} 7\right]=\mathcal{K}_{\sigma(2354)}^{3}-\mathcal{K}_{\sigma(3254)}^{3}+\mathcal{K}_{\sigma(2354)}^{4}-\mathcal{K}_{\sigma(3254)}^{4} \\
& n\left[2_{\sigma} 3_{\sigma}, 1,4_{\sigma}, 6,5_{\sigma} 7\right]=\mathcal{K}_{\sigma(3245)}^{4}-\mathcal{K}_{\sigma(2345)}^{4}-\mathcal{K}_{\sigma(2345)}^{5}+\mathcal{K}_{\sigma(3245)}^{5} \\
& n\left[3_{\sigma} 4_{\sigma}, 5_{\sigma}, 6,2_{\sigma}, 71\right]=\mathcal{K}_{\sigma(2435)}^{1}-\mathcal{K}_{\sigma(2345)}^{1}+\mathcal{K}_{\sigma(2534)}^{1}-\mathcal{K}_{\sigma(2543)}^{1} \\
& n\left[5_{\sigma} 7,1,2_{\sigma}, 6,3_{\sigma} 4_{\sigma}\right]=\mathcal{K}_{\sigma(5234)}^{1}-\mathcal{K}_{\sigma(5243)}^{1}+\mathcal{K}_{\sigma(5234)}^{2}-\mathcal{K}_{\sigma(5243)}^{2} \\
& n\left[5_{\sigma} 7,6,12_{\sigma}, 3_{\sigma} 4_{\sigma}\right]=\mathcal{K}_{\sigma(2435)}^{4}-\mathcal{K}_{\sigma(2345)}^{4}-\mathcal{K}_{\sigma(2345)}^{5}+\mathcal{K}_{\sigma(2435)}^{5} \\
& n\left[67,5_{\sigma}, 1,2_{\sigma}, 3_{\sigma} 4_{\sigma}\right]=\mathcal{K}_{\sigma(2435)}^{5}-\mathcal{K}_{\sigma(2345)}^{5}+\mathcal{K}_{\sigma(3425)}^{5}-\mathcal{K}_{\sigma(4325)}^{5} \\
& n\left[5_{\sigma} 7,4_{\sigma}, 1,2_{\sigma}, 63_{\sigma}\right]=\mathcal{K}_{\sigma(5423)}^{1}+\mathcal{K}_{\sigma(4523)}^{2}+\mathcal{K}_{\sigma(5423)}^{2}+\mathcal{K}_{\sigma(4523)}^{3} \\
& n\left[5_{\sigma} 7,4_{\sigma}, 12_{\sigma}, 3_{\sigma} 6\right]=\mathcal{K}_{\sigma(2543)}^{2}+\mathcal{K}_{\sigma(2453)}^{3}+\mathcal{K}_{\sigma(2543)}^{3}+\mathcal{K}_{\sigma(2453)}^{4} \\
& n\left[2_{\sigma} 3_{\sigma}, 1,6,7,4_{\sigma} 5_{\sigma}\right]=-\mathcal{K}_{\sigma(2345)}^{3}+\mathcal{K}_{\sigma(2354)}^{3}+\mathcal{K}_{\sigma(3245)}^{3}-\mathcal{K}_{\sigma(3254)}^{3} \\
& +\mathcal{K}_{\sigma(2345)}^{5}-\mathcal{K}_{\sigma(2354)}^{5}-\mathcal{K}_{\sigma(3245)}^{5}+\mathcal{K}_{\sigma(3254)}^{5} \\
& n\left[67,1,2_{\sigma} 3_{\sigma}, 4_{\sigma} 5_{\sigma}\right]=+\mathcal{K}_{\sigma(2345)}^{5}-\mathcal{K}_{\sigma(2354)}^{5}-\mathcal{K}_{\sigma(3245)}^{5}+\mathcal{K}_{\sigma(3254)}^{5} \\
& -\mathcal{K}_{\sigma(4523)}^{5}+\mathcal{K}_{\sigma(4532)}^{5}+\mathcal{K}_{\sigma(5423)}^{5}-\mathcal{K}_{\sigma(5432)}^{5} \\
& n\left[12_{\sigma}, 6,3_{\sigma} 4_{\sigma}, 5_{\sigma} 7\right]=-\mathcal{K}_{\sigma(2534)}^{2}+\mathcal{K}_{\sigma(2543)}^{2}-\mathcal{K}_{\sigma(2534)}^{3}+\mathcal{K}_{\sigma(2543)}^{3} \\
& +\mathcal{K}_{\sigma(2345)}^{4}-\mathcal{K}_{\sigma(2435)}^{4}+\mathcal{K}_{\sigma(2345)}^{5}-\mathcal{K}_{\sigma(2435)}^{5} \\
& n\left[2_{\sigma} 3_{\sigma}, 4_{\sigma}, 5_{\sigma}, 6,71\right]=+\mathcal{K}_{\sigma(2345)}^{1}-\mathcal{K}_{\sigma(3245)}^{1}-\mathcal{K}_{\sigma(4235)}^{1}+\mathcal{K}_{\sigma(4325)}^{1} \\
& -\mathcal{K}_{\sigma(5234)}^{1}+\mathcal{K}_{\sigma(5324)}^{1}+\mathcal{K}_{\sigma(5423)}^{1}-\mathcal{K}_{\sigma(5432)}^{1} \\
& n\left[2_{\sigma} 6,1,3_{\sigma}, 7,4_{\sigma} 5_{\sigma}\right]=+\mathcal{K}_{\sigma(4532)}^{1}-\mathcal{K}_{\sigma(5432)}^{1}+\mathcal{K}_{\sigma(3452)}^{2}-\mathcal{K}_{\sigma(3542)}^{2} \\
& -\mathcal{K}_{\sigma(4532)}^{3}+\mathcal{K}_{\sigma(5432)}^{3}-\mathcal{K}_{\sigma(3452)}^{4}+\mathcal{K}_{\sigma(3542)}^{4} \\
& n\left[3_{\sigma} 4_{\sigma}, 5_{\sigma}, 7,1,2_{\sigma} 6\right]=-\mathcal{K}_{\sigma(3452)}^{1}+\mathcal{K}_{\sigma(4352)}^{1}+\mathcal{K}_{\sigma(5342)}^{1}-\mathcal{K}_{\sigma(5432)}^{1} \\
& -\mathcal{K}_{\sigma(3452)}^{4}+\mathcal{K}_{\sigma(4352)}^{4}+\mathcal{K}_{\sigma(5342)}^{4}-\mathcal{K}_{\sigma(5432)}^{4} \\
& n\left[4_{\sigma} 5_{\sigma}, 7,1,6,2_{\sigma} 3_{\sigma}\right]=+\mathcal{K}_{\sigma(4523)}^{1}-\mathcal{K}_{\sigma(4532)}^{1}-\mathcal{K}_{\sigma(5423)}^{1}+\mathcal{K}_{\sigma(5432)}^{1} \\
& -\mathcal{K}_{\sigma(4523)}^{3}+\mathcal{K}_{\sigma(4532)}^{3}+\mathcal{K}_{\sigma(5423)}^{3}-\mathcal{K}_{\sigma(5432)}^{3} \\
& n\left[5_{\sigma} 7,4_{\sigma}, 1,6,2_{\sigma} 3_{\sigma}\right]=+\mathcal{K}_{\sigma(5423)}^{1}-\mathcal{K}_{\sigma(5432)}^{1}+\mathcal{K}_{\sigma(4523)}^{2}-\mathcal{K}_{\sigma(4532)}^{2} \\
& +\mathcal{K}_{\sigma(5423)}^{2}-\mathcal{K}_{\sigma(5432)}^{2}+\mathcal{K}_{\sigma(4523)}^{3}-\mathcal{K}_{\sigma(4532)}^{3} \\
& n\left[67,1,2_{\sigma}, 3_{\sigma}, 4_{\sigma} 5_{\sigma}\right]=+\mathcal{K}_{\sigma(2345)}^{5}-\mathcal{K}_{\sigma(2354)}^{5}-\mathcal{K}_{\sigma(2453)}^{5}+\mathcal{K}_{\sigma(2543)}^{5} \\
& -\mathcal{K}_{\sigma(3452)}^{5}+\mathcal{K}_{\sigma(3542)}^{5}+\mathcal{K}_{\sigma(4532)}^{5}-\mathcal{K}_{\sigma(5432)}^{5} \\
& n\left[71,6,2_{\sigma} 3_{\sigma}, 4_{\sigma} 5_{\sigma}\right]=-\mathcal{K}_{\sigma(2345)}^{1}+\mathcal{K}_{\sigma(2354)}^{1}+\mathcal{K}_{\sigma(3245)}^{1}-\mathcal{K}_{\sigma(3254)}^{1} \\
& +\mathcal{K}_{\sigma(4523)}^{1}-\mathcal{K}_{\sigma(4532)}^{1}-\mathcal{K}_{\sigma(5423)}^{1}+\mathcal{K}_{\sigma(5432)}^{1} \\
& n\left[12_{\sigma}, 6,3_{\sigma}, 4_{\sigma}, 5_{\sigma} 7\right]=+\mathcal{K}_{\sigma(2543)}^{2}+\mathcal{K}_{\sigma(2354)}^{3}+\mathcal{K}_{\sigma(2453)}^{3}+\mathcal{K}_{\sigma(2543)}^{3} \\
& +\mathcal{K}_{\sigma(2345)}^{4}+\mathcal{K}_{\sigma(2354)}^{4}+\mathcal{K}_{\sigma(2453)}^{4}+\mathcal{K}_{\sigma(2345)}^{5} \\
& n\left[12_{\sigma}, 6,3_{\sigma}, 7,4_{\sigma} 5_{\sigma}\right]=+\mathcal{K}_{\sigma(2453)}^{2}-\mathcal{K}_{\sigma(2543)}^{2}+\mathcal{K}_{\sigma(2345)}^{3}-\mathcal{K}_{\sigma(2354)}^{3} \\
& -\mathcal{K}_{\sigma(2453)}^{4}+\mathcal{K}_{\sigma(2543)}^{4}-\mathcal{K}_{\sigma(2345)}^{5}+\mathcal{K}_{\sigma(2354)}^{5} \\
& n\left[2_{\sigma} 3_{\sigma}, 1,6,4_{\sigma}, 5_{\sigma} 7\right]=-\mathcal{K}_{\sigma(2354)}^{3}+\mathcal{K}_{\sigma(3254)}^{3}-\mathcal{K}_{\sigma(2345)}^{4}-\mathcal{K}_{\sigma(2354)}^{4} \\
& +\mathcal{K}_{\sigma(3245)}^{4}+\mathcal{K}_{\sigma(3254)}^{4}-\mathcal{K}_{\sigma(2345)}^{5}+\mathcal{K}_{\sigma(3245)}^{5}
\end{aligned}
$$




$$
\begin{aligned}
& n\left[2_{\sigma} 3_{\sigma}, 4_{\sigma}, 6,1,5_{\sigma} 7\right]=+\mathcal{K}_{\sigma(5234)}^{1}-\mathcal{K}_{\sigma(5324)}^{1}-\mathcal{K}_{\sigma(5423)}^{1}+\mathcal{K}_{\sigma(5432)}^{1} \\
& +\mathcal{K}_{\sigma(5234)}^{2}-\mathcal{K}_{\sigma(5324)}^{2}-\mathcal{K}_{\sigma(5423)}^{2}+\mathcal{K}_{\sigma(5432)}^{2} \\
& n\left[2_{\sigma} 6,1,3_{\sigma} 4_{\sigma}, 5_{\sigma} 7\right]=-\mathcal{K}_{\sigma(5342)}^{1}+\mathcal{K}_{\sigma(5432)}^{1}-\mathcal{K}_{\sigma(5342)}^{2}+\mathcal{K}_{\sigma(5432)}^{2} \\
& +\mathcal{K}_{\sigma(3452)}^{3}-\mathcal{K}_{\sigma(4352)}^{3}+\mathcal{K}_{\sigma(3452)}^{4}-\mathcal{K}_{\sigma(4352)}^{4} \\
& n\left[3_{\sigma} 4_{\sigma}, 5_{\sigma}, 7,6,12_{\sigma}\right]=-\mathcal{K}_{\sigma(2345)}^{2}+\mathcal{K}_{\sigma(2435)}^{2}+\mathcal{K}_{\sigma(2534)}^{2}-\mathcal{K}_{\sigma(2543)}^{2} \\
& -\mathcal{K}_{\sigma(2345)}^{5}+\mathcal{K}_{\sigma(2435)}^{5}+\mathcal{K}_{\sigma(2534)}^{5}-\mathcal{K}_{\sigma(2543)}^{5} \\
& n\left[5_{\sigma} 7,6,1,2_{\sigma}, 3_{\sigma} 4_{\sigma}\right]=-\mathcal{K}_{\sigma(2345)}^{4}+\mathcal{K}_{\sigma(2435)}^{4}+\mathcal{K}_{\sigma(3425)}^{4}-\mathcal{K}_{\sigma(4325)}^{4} \\
& -\mathcal{K}_{\sigma(2345)}^{5}+\mathcal{K}_{\sigma(2435)}^{5}+\mathcal{K}_{\sigma(3425)}^{5}-\mathcal{K}_{\sigma(4325)}^{5} \\
& n\left[2_{\sigma} 6,1,3_{\sigma}, 4_{\sigma}, 5_{\sigma} 7\right]=+\mathcal{K}_{\sigma(5432)}^{1}+\mathcal{K}_{\sigma(3542)}^{2}+\mathcal{K}_{\sigma(4532)}^{2}+\mathcal{K}_{\sigma(5432)}^{2} \\
& +\mathcal{K}_{\sigma(3452)}^{3}+\mathcal{K}_{\sigma(3542)}^{3}+\mathcal{K}_{\sigma(4532)}^{3}+\mathcal{K}_{\sigma(3452)}^{4} \\
& n\left[4_{\sigma} 5_{\sigma}, 7,16,2_{\sigma} 3_{\sigma}\right]=+\mathcal{K}_{\sigma(4523)}^{1}-\mathcal{K}_{\sigma(4532)}^{1}-\mathcal{K}_{\sigma(5423)}^{1}+\mathcal{K}_{\sigma(5432)}^{1}-\mathcal{K}_{\sigma(3245)}^{5}+\mathcal{K}_{\sigma(3254)}^{5} \\
& -\mathcal{K}_{\sigma(2345)}^{3}+\mathcal{K}_{\sigma(2354)}^{3}+\mathcal{K}_{\sigma(3245)}^{3}-\mathcal{K}_{\sigma(3254)}^{3}-\mathcal{K}_{\sigma(4523)}^{3}+\mathcal{K}_{\sigma(4532)}^{3} \\
& +\mathcal{K}_{\sigma(5423)}^{3}-\mathcal{K}_{\sigma(5432)}^{3}+\mathcal{K}_{\sigma(2345)}^{5}-\mathcal{K}_{\sigma(2354)}^{5} \\
& n\left[16,2_{\sigma}, 3_{\sigma} 4_{\sigma}, 5_{\sigma} 7\right]=-\mathcal{K}_{\sigma(5342)}^{1}+\mathcal{K}_{\sigma(5432)}^{1}-\mathcal{K}_{\sigma(2534)}^{2}+\mathcal{K}_{\sigma(2543)}^{2}+\mathcal{K}_{\sigma(2345)}^{5}-\mathcal{K}_{\sigma(2435)}^{5} \\
& -\mathcal{K}_{\sigma(5342)}^{2}+\mathcal{K}_{\sigma(5432)}^{2}-\mathcal{K}_{\sigma(2534)}^{3}+\mathcal{K}_{\sigma(2543)}^{3}+\mathcal{K}_{\sigma(3452)}^{3}-\mathcal{K}_{\sigma(4352)}^{3} \\
& +\mathcal{K}_{\sigma(2345)}^{4}-\mathcal{K}_{\sigma(2435)}^{4}+\mathcal{K}_{\sigma(3452)}^{4}-\mathcal{K}_{\sigma(4352)}^{4} \\
& n\left[16,7,2_{\sigma} 3_{\sigma}, 4_{\sigma} 5_{\sigma}\right]=+\mathcal{K}_{\sigma(2345)}^{1}-\mathcal{K}_{\sigma(2354)}^{1}-\mathcal{K}_{\sigma(3245)}^{1}+\mathcal{K}_{\sigma(3254)}^{1}-\mathcal{K}_{\sigma(5423)}^{5}+\mathcal{K}_{\sigma(5432)}^{5} \\
& -\mathcal{K}_{\sigma(4523)}^{1}+\mathcal{K}_{\sigma(4532)}^{1}+\mathcal{K}_{\sigma(5423)}^{1}-\mathcal{K}_{\sigma(5432)}^{1}-\mathcal{K}_{\sigma(2345)}^{5}+\mathcal{K}_{\sigma(2354)}^{5} \\
& +\mathcal{K}_{\sigma(3245)}^{5}-\mathcal{K}_{\sigma(3254)}^{5}+\mathcal{K}_{\sigma(4523)}^{5}-\mathcal{K}_{\sigma(4532)}^{5} \\
& n\left[2_{\sigma} 3_{\sigma}, 4_{\sigma}, 5_{\sigma}, 7,16\right]=-\mathcal{K}_{\sigma(2345)}^{1}+\mathcal{K}_{\sigma(3245)}^{1}+\mathcal{K}_{\sigma(4235)}^{1}-\mathcal{K}_{\sigma(4325)}^{1}+\mathcal{K}_{\sigma(5423)}^{5}-\mathcal{K}_{\sigma(5432)}^{5} \\
& +\mathcal{K}_{\sigma(5234)}^{1}-\mathcal{K}_{\sigma(5324)}^{1}-\mathcal{K}_{\sigma(5423)}^{1}+\mathcal{K}_{\sigma(5432)}^{1}+\mathcal{K}_{\sigma(2345)}^{5}-\mathcal{K}_{\sigma(3245)}^{5} \\
& -\mathcal{K}_{\sigma(4235)}^{5}+\mathcal{K}_{\sigma(4325)}^{5}-\mathcal{K}_{\sigma(5234)}^{5}+\mathcal{K}_{\sigma(5324)}^{5} \\
& n\left[2_{\sigma} 3_{\sigma}, 4_{\sigma}, 5_{\sigma} 7,16\right]=+\mathcal{K}_{\sigma(5234)}^{1}-\mathcal{K}_{\sigma(5324)}^{1}-\mathcal{K}_{\sigma(5423)}^{1}+\mathcal{K}_{\sigma(5432)}^{1}-\mathcal{K}_{\sigma(4235)}^{5}+\mathcal{K}_{\sigma(4325)}^{5} \\
& +\mathcal{K}_{\sigma(5234)}^{2}-\mathcal{K}_{\sigma(5324)}^{2}-\mathcal{K}_{\sigma(5423)}^{2}+\mathcal{K}_{\sigma(5432)}^{2}+\mathcal{K}_{\sigma(2345)}^{4}-\mathcal{K}_{\sigma(3245)}^{4} \\
& -\mathcal{K}_{\sigma(4235)}^{4}+\mathcal{K}_{\sigma(4325)}^{4}+\mathcal{K}_{\sigma(2345)}^{5}-\mathcal{K}_{\sigma(3245)}^{5} \\
& n\left[16,2_{\sigma}, 3_{\sigma}, 4_{\sigma}, 5_{\sigma} 7\right]=+\mathcal{K}_{\sigma(5432)}^{1}+\mathcal{K}_{\sigma(2543)}^{2}+\mathcal{K}_{\sigma(3542)}^{2}+\mathcal{K}_{\sigma(4532)}^{2}+\mathcal{K}_{\sigma(3452)}^{4}+\mathcal{K}_{\sigma(2345)}^{5} \\
& +\mathcal{K}_{\sigma(5432)}^{2}+\mathcal{K}_{\sigma(2354)}^{3}+\mathcal{K}_{\sigma(2453)}^{3}+\mathcal{K}_{\sigma(2543)}^{3}+\mathcal{K}_{\sigma(3452)}^{3}+\mathcal{K}_{\sigma(3542)}^{3} \\
& +\mathcal{K}_{\sigma(4532)}^{3}+\mathcal{K}_{\sigma(2345)}^{4}+\mathcal{K}_{\sigma(2354)}^{4}+\mathcal{K}_{\sigma(2453)}^{4} \\
& n\left[16,2_{\sigma}, 3_{\sigma}, 7,4_{\sigma} 5_{\sigma}\right]=+\mathcal{K}_{\sigma(4532)}^{1}-\mathcal{K}_{\sigma(5432)}^{1}+\mathcal{K}_{\sigma(2453)}^{2}-\mathcal{K}_{\sigma(2543)}^{2}-\mathcal{K}_{\sigma(2345)}^{5}+\mathcal{K}_{\sigma(2354)}^{5} \\
& +\mathcal{K}_{\sigma(3452)}^{2}-\mathcal{K}_{\sigma(3542)}^{2}+\mathcal{K}_{\sigma(2345)}^{3}-\mathcal{K}_{\sigma(2354)}^{3}-\mathcal{K}_{\sigma(4532)}^{3}+\mathcal{K}_{\sigma(5432)}^{3} \\
& -\mathcal{K}_{\sigma(2453)}^{4}+\mathcal{K}_{\sigma(2543)}^{4}-\mathcal{K}_{\sigma(3452)}^{4}+\mathcal{K}_{\sigma(3542)}^{4} \\
& n\left[3_{\sigma} 4_{\sigma}, 5_{\sigma}, 7,2_{\sigma}, 16\right]=-\mathcal{K}_{\sigma(3452)}^{1}+\mathcal{K}_{\sigma(4352)}^{1}+\mathcal{K}_{\sigma(5342)}^{1}-\mathcal{K}_{\sigma(5432)}^{1}+\mathcal{K}_{\sigma(2534)}^{5}-\mathcal{K}_{\sigma(2543)}^{5} \\
& -\mathcal{K}_{\sigma(2345)}^{2}+\mathcal{K}_{\sigma(2435)}^{2}+\mathcal{K}_{\sigma(2534)}^{2}-\mathcal{K}_{\sigma(2543)}^{2}-\mathcal{K}_{\sigma(3452)}^{4}+\mathcal{K}_{\sigma(4352)}^{4} \\
& +\mathcal{K}_{\sigma(5342)}^{4}-\mathcal{K}_{\sigma(5432)}^{4}-\mathcal{K}_{\sigma(2345)}^{5}+\mathcal{K}_{\sigma(2435)}^{5}
\end{aligned}
$$




$$
\begin{aligned}
n\left[5_{\sigma} 7,4_{\sigma}, 16,2_{\sigma} 3_{\sigma}\right]= & +\mathcal{K}_{\sigma(5423)}^{1}-\mathcal{K}_{\sigma(5432)}^{1}+\mathcal{K}_{\sigma(4523)}^{2}-\mathcal{K}_{\sigma(4532)}^{2}-\mathcal{K}_{\sigma(2345)}^{5}+\mathcal{K}_{\sigma(3245)}^{5} \\
& +\mathcal{K}_{\sigma(5423)}^{2}-\mathcal{K}_{\sigma(5432)}^{2}-\mathcal{K}_{\sigma(2354)}^{3}+\mathcal{K}_{\sigma(3254)}^{3}+\mathcal{K}_{\sigma(4523)}^{3}-\mathcal{K}_{\sigma(4532)}^{3} \\
& -\mathcal{K}_{\sigma(2345)}^{4}-\mathcal{K}_{\sigma(2354)}^{4}+\mathcal{K}_{\sigma(3245)}^{4}+\mathcal{K}_{\sigma(3254)}^{4}
\end{aligned}
$$

They follow from comparing the field theory limit of $\mathcal{A}_{7}^{\text {string }}\left(1,2_{\rho}, 3_{\rho}, 4_{\rho}, 5_{\rho}, 6_{\rho}, 7\right)$ with

$$
\begin{aligned}
& \mathcal{A}_{7}\left(1,2_{\rho}, 3_{\rho}, 4_{\rho}, 5_{\rho}, 6_{\rho}, 7\right)=\frac{n\left[12_{\rho}, 3_{\rho}, 4_{\rho} 5_{\rho}, 6_{\rho} 7\right]}{s_{12_{\rho}} s_{12_{\rho} 3_{\rho}} s_{4_{\rho}} 5_{\rho} s_{6} 7}-\frac{n\left[2_{\rho} 3_{\rho}, 1,4_{\rho} 5_{\rho}, 6_{\rho} 7\right]}{s_{2} 3_{\rho} s_{12} 3_{\rho} s_{4_{\rho} 5_{\rho}} s_{6} 7}
\end{aligned}
$$

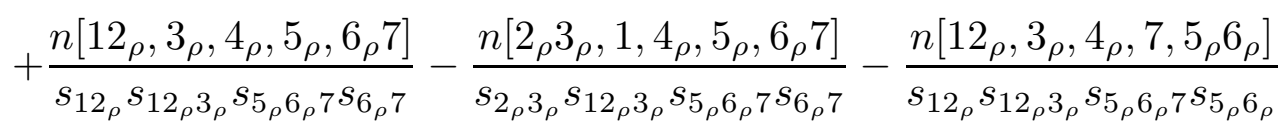

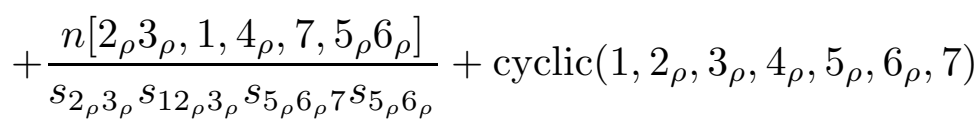

\section{Appendix C. Component expressions at four-point}

This appendix gives an example how the supersymmetric expressions for BCJ numerators decompose in components. In general, component expansion spoils the simplicity of the pure spinor superspace results; but they can be done [21]. At five point level, for instance, the innocent-looking numerator $\left\langle T_{12} T_{34} V_{5}\right\rangle$ contributes $\sim 100$ terms to the five gluon amplitude. That is why we give no more than the four point building blocks $n_{s}=\left\langle T_{12} V_{3} V_{4}\right\rangle=\left\langle V_{1} V_{2} T_{34}\right\rangle$.

There are four inequivalent bose-fermi combinations to consider, namely

$$
(1,2,3,4) \in\{(b, b, b, b),(f, f, b, b),(b, f, b, f),(f, f, f, f)\} .
$$

The numerator then evaluates [21] to

$$
\begin{gathered}
\left.2880 n_{s}\right|_{b b b b}=-\left(k^{1} \cdot e^{2}\right)\left(k^{1} \cdot e^{4}\right)\left(e^{1} \cdot e^{3}\right)-\left(k^{1} \cdot e^{2}\right)\left(k^{2} \cdot e^{4}\right)\left(e^{1} \cdot e^{3}\right) \\
+\left(k^{1} \cdot e^{2}\right)\left(k^{4} \cdot e^{1}\right)\left(e^{3} \cdot e^{4}\right)-\left(k^{1} \cdot e^{2}\right)\left(k^{4} \cdot e^{3}\right)\left(e^{1} \cdot e^{4}\right)+\left(k^{1} \cdot e^{3}\right)\left(k^{1} \cdot e^{4}\right)\left(e^{1} \cdot e^{2}\right) \\
+\left(k^{1} \cdot e^{3}\right)\left(k^{2} \cdot e^{4}\right)\left(e^{1} \cdot e^{2}\right)+\left(k^{1} \cdot e^{4}\right)\left(k^{2} \cdot e^{1}\right)\left(e^{2} \cdot e^{3}\right)+\left(k^{1} \cdot e^{4}\right)\left(k^{4} \cdot e^{3}\right)\left(e^{1} \cdot e^{2}\right) \\
+\left(k^{2} \cdot e^{1}\right)\left(k^{2} \cdot e^{4}\right)\left(e^{2} \cdot e^{3}\right)-\left(k^{2} \cdot e^{1}\right)\left(k^{4} \cdot e^{2}\right)\left(e^{3} \cdot e^{4}\right)+\left(k^{2} \cdot e^{1}\right)\left(k^{4} \cdot e^{3}\right)\left(e^{2} \cdot e^{4}\right) \\
+\frac{1}{4}\left(s_{14}-s_{13}\right)\left(e^{1} \cdot e^{2}\right)\left(e^{3} \cdot e^{4}\right)+\frac{s_{12}}{4}\left[\left(e^{1} \cdot e^{4}\right)\left(e^{2} \cdot e^{3}\right)-\left(e^{1} \cdot e^{3}\right)\left(e^{2} \cdot e^{4}\right)\right] \\
\left.2880 n_{s}\right|_{f f b b}=\left(\chi^{1} \gamma^{e^{3}} \chi^{2}\right)\left(k^{1} \cdot e^{4}\right)-\left(\chi^{1} \gamma^{k^{4}} \chi^{2}\right)\left(e^{3} \cdot e^{4}\right)+\left(\chi^{1} \gamma^{e^{3}} \chi^{2}\right)\left(k^{2} \cdot e^{4}\right)+\left(\chi^{1} \gamma^{e^{4}} \chi^{2}\right)\left(k^{4} \cdot e^{3}\right) \\
\left.2880 n_{s}\right|_{b f b f}=\frac{1}{2}\left[\left(\chi^{2} \gamma^{k^{1} e^{1} e^{3}} \chi^{4}\right)+\left(\chi^{2} \gamma^{k^{1}} \chi^{4}\right)\left(e^{1} \cdot e^{3}\right)-\left(\chi^{2} \gamma^{e^{1}} \chi^{4}\right)\left(k^{1} \cdot e^{3}\right)\right]-\left(\chi^{2} \gamma^{e^{3}} \chi^{4}\right)\left(k^{2} \cdot e^{1}\right) \\
\left.2880 n_{s}\right|_{f f f f}=\left(\chi^{1} \gamma^{m} \chi^{2}\right)\left(\chi^{3} \gamma_{m} \chi^{4}\right)
\end{gathered}
$$

where the SCHOONSCHIP notation has been used, i.e., $\left(\chi^{1} \gamma^{m} \chi^{3}\right) e_{m}^{2} \equiv\left(\chi^{1} \gamma^{e^{2}} \chi^{3}\right)$ as well as $\left(\chi^{2} \gamma^{m n p} \chi^{4}\right) k_{m}^{1} e_{n}^{1} e_{p}^{3} \equiv\left(\chi^{2} \gamma^{k^{1} e^{1} e^{3}} \chi^{4}\right)$. The zero mode integrations involved in the four-point calculations have been performed in [19] for the first time. 


\section{Appendix D. Spinor helicity evaluation at five-point}

In this appendix, we will express the BCJ numerators of a five gluon MHV amplitude in terms of spinor helicity variables. This is meant as a sample calculation showing the relevance of our methods for $D=4$ physics.

Doing so requires running the program [21] for expanding $\left\langle T_{i j} T_{k l} V_{m}\right\rangle$ and $\left\langle T_{i j k} V_{l} V_{m}\right\rangle$ in superfield components, discarding all fermionic contributions. As we have emphasized in the previous appendix, evaluation in components spoils the compactness of the superspace expressions: Each of the brackets above contain several terms with products of polarization vectors and momenta before plugging in the helicity specific spinor products.

If the helicities of the gluons are $(--+++)$ we use the following conventions,

$$
e_{\alpha \dot{\alpha}}^{I}=\sqrt{2} \frac{\psi_{\alpha}^{I} \bar{\chi}_{\dot{\alpha}}^{I}}{\left[\bar{\psi}^{I} \bar{\chi}^{I}\right]}, \quad I=1,2, \quad e_{\dot{\beta} \beta}^{J}=\sqrt{2} \frac{\bar{\psi}_{\dot{\beta}}^{J} \chi_{\beta}^{J}}{\left\langle\chi^{J} \psi^{J}\right\rangle}, \quad J=3,4,5
$$

where $\langle\psi \chi\rangle=\psi^{\alpha} \chi_{\alpha}=\epsilon^{\alpha \beta} \psi_{\beta} \chi_{\alpha}$ and $[\bar{\psi} \bar{\chi}]=\bar{\psi}_{\dot{\alpha}} \bar{\chi}^{\dot{\alpha}}=\epsilon_{\dot{\alpha} \dot{\beta}} \bar{\psi}_{\dot{\beta}} \bar{\chi}^{\dot{\alpha}}$ are the spinor products and $\langle i j\rangle[i j]=-2 s_{i j}$. For the specific choice $(2,1,1,1,1)$ of reference momenta $\chi_{\alpha}^{J}, \bar{\chi}_{\dot{\alpha}}^{I}$ they imply

$$
\begin{gathered}
\left(e^{1} \cdot e^{3}\right)=\left(e^{1} \cdot e^{4}\right)=\left(e^{1} \cdot e^{5}\right)=\left(e^{3} \cdot e^{4}\right)=\left(e^{3} \cdot e^{5}\right)=\left(e^{4} \cdot e^{5}\right)=0 \\
\left(k^{2} \cdot e^{1}\right)=\left(k^{1} \cdot e^{2}\right)=\left(k^{1} \cdot e^{3}\right)=\left(k^{1} \cdot e^{4}\right)=\left(k^{1} \cdot e^{5}\right)=0
\end{gathered}
$$

and we quickly obtain the following basis kinematics (dropping an overall numerical coefficient)

$$
\begin{aligned}
& \mathcal{K}_{(23)}^{1}=\frac{\langle 12\rangle^{3}[25]^{2}[43]}{[12]\langle 13\rangle\langle 14\rangle}, \quad \mathcal{K}_{(32)}^{1}=\frac{\langle 12\rangle^{3}[24][25][53]}{[12]\langle 13\rangle\langle 14\rangle}, \quad \mathcal{K}_{(23)}^{2}=0 \\
& \mathcal{K}_{(32)}^{2}=\frac{\langle 12\rangle^{3}[23][24][35]}{[12]\langle 14\rangle\langle 51\rangle}, \quad \mathcal{K}_{(23)}^{3}=0, \quad \mathcal{K}_{(32)}^{3}=\frac{\langle 12\rangle^{3}[23]^{2}[45]}{[12]\langle 14\rangle\langle 15\rangle}
\end{aligned}
$$

which translates into BCJ numerators $n_{1}=n_{3}=n_{12}=0$ and

$$
\begin{gathered}
\left(n_{2}, n_{4}, n_{5}, n_{6}, n_{7}, n_{8}\right)=\frac{\langle 12\rangle^{3}}{[12]} \\
\times\left(\frac{[23][25][45]}{\langle 13\rangle\langle 14\rangle}, \frac{[23]^{2}[45]}{\langle 14\rangle\langle 51\rangle}, \frac{[25]^{2}[43]}{\langle 13\rangle\langle 14\rangle}, \frac{[24][25][34]}{\langle 13\rangle\langle 15\rangle}, \frac{[23][24][45]}{\langle 13\rangle\langle 51\rangle}, \frac{[25]^{2}[43]}{\langle 13\rangle\langle 14\rangle}\right) \\
\left(n_{9}, n_{10}, n_{11}, n_{13}, n_{14}, n_{15}\right)=\frac{\langle 12\rangle^{3}}{[12]} \\
\times\left(\frac{[23][25][34]}{\langle 14\rangle\langle 51\rangle}, \frac{[23][24][35]}{\langle 14\rangle\langle 51\rangle}, \frac{[24][25][35]}{\langle 13\rangle\langle 14\rangle}, \frac{[24]^{2}[35]}{\langle 13\rangle\langle 51\rangle}, \frac{[24]^{2}[35]}{\langle 13\rangle\langle 15\rangle}, \frac{[23]^{2}[45]}{\langle 14\rangle\langle 15\rangle}\right)
\end{gathered}
$$


They can be easily checked to reproduce the Parke-Taylor formula [32,33].

All the nonlocalities $\sim[12]^{-1},\langle 13\rangle^{-1},\langle 14\rangle^{-1},\langle 15\rangle^{-1}$ are spurious and arise from the reference momentum dependent denominators of (D.1). As we have emphasized, all the $n_{i}$ in this work are local in any spacetime dimension. 


\section{References}

[1] Z. Bern, J.J.M. Carrasco, H. Johansson, "New Relations for Gauge-Theory Amplitudes," Phys. Rev. D78, 085011 (2008). [arXiv:0805.3993 [hep-ph]].

[2] Z. Bern, J.J.M. Carrasco, H. Johansson, "Perturbative Quantum Gravity as a Double Copy of Gauge Theory," Phys. Rev. Lett. 105, 061602 (2010). [arXiv:1004.0476 [hepth]].

[3] M. Kiermaier, Amplitudes 2010, Queen Mary, University of London, http://www.strings.ph.qmul.ac.uk/ theory/Amplitudes2010/Talks/MK2010.pdf

[4] N.E.J. Bjerrum-Bohr, P.H. Damgaard, T. Sondergaard and P. Vanhove, "The Momentum Kernel of Gauge and Gravity Theories," JHEP 1101, 001 (2011) [arXiv:1010.3933 [hep-th]].

[5] C.R. Mafra, O. Schlotterer and S. Stieberger, to appear

[6] C.R. Mafra, "Simplifying the Tree-level Superstring Massless Five-point Amplitude," JHEP 1001, 007 (2010) [arXiv:0909.5206 [hep-th]].

[7] N.E.J. Bjerrum-Bohr, P.H. Damgaard, T. Sondergaard, P. Vanhove, "Monodromy and Jacobi-like Relations for Color-Ordered Amplitudes," JHEP 1006, 003 (2010). [arXiv:1003.2403 [hep-th]].

[8] Z. Bern, J. J. Carrasco, L. J. Dixon, H. Johansson, R. Roiban, "Amplitudes and Ultraviolet Behavior of N=8 Supergravity," [arXiv:1103.1848 [hep-th]].

[9] N. Berkovits, "Super-Poincare covariant quantization of the superstring," JHEP 0004, 018 (2000) [arXiv:hep-th/0001035].

[10] N. Berkovits, "ICTP lectures on covariant quantization of the superstring," [hepth/0209059].

[11] O.A. Bedoya, N. Berkovits, "GGI Lectures on the Pure Spinor Formalism of the Superstring," [arXiv:0910.2254 [hep-th]].

[12] E. Witten, "Twistor - Like Transform In Ten-Dimensions," Nucl. Phys. B 266, 245 (1986).

[13] W. Siegel, "Classical Superstring Mechanics," Nucl. Phys. B263, 93 (1986).

[14] P.S. Howe, "Pure spinors lines in superspace and ten-dimensional supersymmetric theories," Phys. Lett. B258, 141-144 (1991).

[15] R. Medina, F.T. Brandt and F.R. Machado, "The open superstring 5-point amplitude revisited," JHEP 0207, 071 (2002) [arXiv:hep-th/0208121] ;

L.A. Barreiro and R. Medina, "5-field terms in the open superstring effective action," JHEP 0503, 055 (2005) [arXiv:hep-th/0503182].

[16] D. Oprisa and S. Stieberger, "Six gluon open superstring disk amplitude, multiple hypergeometric series and Euler-Zagier sums," arXiv:hep-th/0509042.

[17] S. Stieberger, T.R. Taylor, "Amplitude for N-Gluon Superstring Scattering," Phys. Rev. Lett. 97, 211601 (2006). [hep-th/0607184]; "Multi-Gluon Scattering in Open 
Superstring Theory," Phys. Rev. D74, 126007 (2006). [hep-th/0609175]; "Supersymmetry Relations and MHV Amplitudes in Superstring Theory," Nucl. Phys. B793, 83-113 (2008). [arXiv:0708.0574 [hep-th]]; "Complete Six-Gluon Disk Amplitude in Superstring Theory," Nucl. Phys. B801, 128-152 (2008). [arXiv:0711.4354 [hep-th]].

[18] J.P. Harnad and S. Shnider, "Constraints And Field Equations For Ten-Dimensional Superyang-Mills Theory," Commun. Math. Phys. 106, 183 (1986) ;

P.A. Grassi and L. Tamassia, "Vertex operators for closed superstrings," JHEP 0407, 071 (2004) [arXiv:hep-th/0405072].

[19] G. Policastro and D. Tsimpis, " $R^{4}$, purified," Class. Quant. Grav. 23, 4753 (2006) [arXiv:hep-th/0603165].

[20] J.A.M. Vermaseren, "New features of FORM," arXiv:math-ph/0010025. ;

M. Tentyukov and J.A.M. Vermaseren, "The multithreaded version of FORM," arXiv:hep-ph/0702279.

[21] C.R. Mafra, "PSS: A FORM Program to Evaluate Pure Spinor Superspace Expressions," [arXiv:1007.4999 [hep-th]].

[22] C.R. Mafra, "Pure Spinor Superspace Identities for Massless Four-point Kinematic Factors," JHEP 0804, 093 (2008). [arXiv:0801.0580 [hep-th]].

[23] C.R. Mafra, "Towards Field Theory Amplitudes From the Cohomology of Pure Spinor Superspace," JHEP 1011, 096 (2010) [arXiv:1007.3639 [hep-th]].

[24] C.R. Mafra, O. Schlotterer, S. Stieberger and D. Tsimpis, "A recursive formula for N-point SYM tree amplitudes," arXiv:1012.3981 [hep-th].

[25] R. Kleiss, H. Kuijf, "Multi - Gluon Cross-sections And Five Jet Production At Hadron Colliders," Nucl. Phys. B312, 616 (1989).

[26] C.R. Mafra, O. Schlotterer, S. Stieberger and D. Tsimpis, "Six Open String Disk Amplitude in Pure Spinor Superspace," Nucl. Phys. B 846, 359 (2011) [arXiv:1011.0994 [hep-th]].

[27] N.E.J. Bjerrum-Bohr, P.H. Damgaard, P. Vanhove, "Minimal Basis for Gauge Theory Amplitudes," Phys. Rev. Lett. 103, 161602 (2009). [arXiv:0907.1425 [hep-th]].

[28] S. Stieberger, "Open \& Closed vs. Pure Open String Disk Amplitudes," [arXiv:0907.2211 [hep-th]].

[29] S.H. Henry Tye and Y. Zhang, "Dual Identities inside the Gluon and the Graviton Scattering Amplitudes," JHEP 1006, 071 (2010) [arXiv:1003.1732 [hep-th]].

[30] D. Vaman, Y.-P. Yao, "Constraints and Generalized Gauge Transformations on TreeLevel Gluon and Graviton Amplitudes," JHEP 1011, 028 (2010). [arXiv:1007.3475 [hep-th]].

[31] N. Arkani-Hamed, F. Cachazo, C. Cheung, J. Kaplan, "The S-Matrix in Twistor Space," JHEP 1003, 110 (2010). [arXiv:0903.2110 [hep-th]].

[32] S.J. Parke, T.R. Taylor, "An Amplitude for $n$ Gluon Scattering," Phys. Rev. Lett. 56, 2459 (1986).

[33] F.A. Berends, W.T. Giele, "Recursive Calculations for Processes with n Gluons," Nucl. Phys. B306, 759 (1988). 